\title{
Simulation system of spinal cord motor nuclei and associated nerves and muscles, in a Web-based architecture
}

\author{
Rogerio R. L. Cisi • André F. Kohn
}

Received: 6 December 2007 /Revised: 4 March 2008 / Accepted: 17 March 2008 / Published online: 28 May 2008

(C) Springer Science + Business Media, LLC 2008

\begin{abstract}
A Web-based simulation system of the spinal cord circuitry responsible for muscle control is described. The simulator employs two-compartment motoneuron models for S, FR and FF types, with synaptic inputs acting through conductance variations. Four motoneuron pools with their associated interneurons are represented in the simulator, with the possibility of inclusion of more than 2,000 neurons and 2,000,000 synapses. Each motoneuron action potential is followed, after a conduction delay, by a motor unit potential and a motor unit twitch. The sums of all motor unit potentials and twitches result in the electromyogram (EMG), and the muscle force, respectively. Inputs to the motoneuron pool come from populations of interneurons (Ia reciprocal inhibitory interneurons, Ib interneurons, and Renshaw cells) and from stochastic point processes associated with descending tracts. To simulate human electrophysiological experiments, the simulator incorporates external nerve stimulation with orthodromic and antidromic propagation. This provides the mechanisms for reflex generation and activation of spinal neuronal circuits that modulate the activity of another motoneuron pool (e.g., by reciprocal inhibition). The generation of the Hreflex by the Ia-motoneuron pool system and its modulation by spinal cord interneurons is included in the simulation system. Studies with the simulator may include the statistics of individual motoneuron or interneuron spike trains or the collective effect of a motor nucleus on the dynamics of
\end{abstract}

Action Editor: Eberhard Fetz

R. R. L. Cisi $(\bowtie) \cdot$ A. F. Kohn $(\bowtie)$

Biomedical Engineering Laboratory, Escola Politécnica,

Universidade de São Paulo,

São Paulo, Brazil

e-mail: rcisi@leb.usp.br

e-mail: andfkohn@leb.usp.br muscle force control. Properties associated with motor-unit recruitment, motor-unit synchronization, recurrent inhibition and reciprocal inhibition may be investigated.

Keywords Motoneuron · Interneuron · Renshaw cell · Neuronal network $\cdot$ Motoneuron pool $\cdot$ Muscle $\cdot$ Force . $\mathrm{EMG} \cdot \mathrm{H}-\mathrm{reflex} \cdot$ Modeling $\cdot$ Simulator

\section{Introduction}

The spinal cord circuitry is one of the most investigated neural systems, formed by complex networks of motoneurons, interneurons, sensory afferents and descending tracts. The spinal cord ventral horn contains the motor nuclei that drive the muscles of the limbs and trunk, whereas the dorsal horn receives sensory information from the body, which is distributed synaptically to spinal cord motoneurons, interneurons and supraspinal nuclei.

Within the context of motor control, motoneurons (MN) are central elements in the spinal circuitry, responsible for the integration of central and peripheral inputs, in order to activate muscle fibers. A single alpha motoneuron and all of the corresponding muscle fibers it innervates constitutes a motor-unit (MU). According to their physiological properties, motor-units are generally classified as slow twitch (S type), fast twitch, fatigue resistant (FR type) and fast twitch, fast fatiguing (FF type), as proposed by Burke et al. (1973). The motoneurons in this paper will be modelled in either of the three classes S, FR and FF.

Interneurons (IN) also play an important role in the spinal cord motor control. Renshaw cells (RC) are interneurons that inhibit the parent and other motoneurons in the vicinity, acting in the so-called recurrent inhibition pathway. One function of the Ia inhibitory interneurons 
(IaIn) is making reciprocal inhibition between antagonist motoneuron pools, besides other tasks. Ib inhibitory interneurons (IbIn) are mainly involved with inhibition from Ib homonymous afferents to a otoneuron pool.

Other neuronal structures take part in the spinal cord motor control, such as sensory fibers and descending tracts from the brain. The former transmit proprioceptive and cutaneous information from the muscles and the body to the spinal cord neuronal circuitry, while the latter drive the motoneurons and interneurons. Reviews on spinal cord circuitry may be found in Burke (2004) and PierrotDeseilligny and Burke (2005).

The investigation of human neurophysiology is mostly limited to non-invasive approaches, due to ethical considerations. Despite technological advances in experimental techniques, the results from human experiments frequently raise many hypotheses, usually impossible to be tested in humans. Computer simulations can help in the hypothesis testing by providing several procedures and measurements not available experimentally. Simulations are also useful for theoretical investigations of neural systems properties, for demonstrating rules and paradigms in neuroscience and for the study of neuropathology.

General-purpose simulators have been used in computational neuroscience (Bower et al. 2003; Carnevale and Hines 2006), conveying information from ion channels to neuronal networks. However, some level of customization is required with the intention of performing complex simulations or in the development of a simulation system. On the other hand, more focused approaches have been adopted by research groups interested in answering specific questions in spinal cord investigation, which meant developing their own simulators, without a goal of general usage (Capaday and Stein 1987; Maltenfort et al. 1998; Nussbaumer et al. 2002; Ivashko et al. 2003; Lowery and Erim 2005; Subramanian et al. 2005; Stienen et al. 2007; Uchiyama and Windhorst 2007). Bashor's (1998) spinal network simulator was based on MacGregor's implementations in Fortran of neuronal, synaptic and network models (MacGregor 1987). Its focus was on the interactions between the neuronal networks associated with two antagonistic muscles, with parameter values based on cat data from the literature. It assumes all 169 motoneurons of a given pool are identical. A very detailed interneuronal network contributes to the modulation of the motoneurons' spike trains. Lowery and Erim (2005) presented a simulation study of the effects of common oscillatory inputs on the correlated discharges of a pool of 100 motor units. The input command was realized by means of current injected in the soma and the outputs were muscle force and the spike trains of the motor-units. Subramanian et al. (2005) expanded the model developed by Bashor (1998) with powerful visualization tools. It combines detailed anatom- ical and physiological aspects of the cat spinal cord. Stienen et al. (2007) studied muscle reflex modulation by putting together the spinal cord neuronal network model of Bashor (1998) with a simple one-degree-of-freedom muscle-skeletal model. Stienen et al. (2007) compute the average output over the motoneuron pool which is then smoothed to provide the input to a mass-spring-damper system. They used simulator outputs to propose neural mechanisms for human neuropathological observations. Uchiyama and Windhorst (2007) studied the effects of Renshaw cell recurrent inhibition on computer-simulated cat medial gastrocnemius motoneurons (250 to 300$)$. The simulator differentiated motoneurons by types and the focus was to quantify the degree of motoneuron synchronization as a function of Renshaw cell feedback inhibition.

The simulator described here employs differentiated motoneuron models ( $\mathrm{S}, \mathrm{FR}$ and $\mathrm{FF}$ ), with selectable numbers of units. Each motoneuron generates, after a conduction delay, a motor-unit potential and a motor-unit twitch. The sum of the latter over all the motor-units results in the total muscle force. The superposition of the former over all the motor-units gives the muscle electromyogram. Inputs to the motoneuron pool come from populations of interneurons (IaIN, IbIN and Renshaw cells) and from stochastic point processes associated with descending tracts. To simulate electrophysiological experiments in humans, the simulator incorporates external nerve stimulation with orthodromic and antidromic propagation. This provides the mechanisms for reflex generation and activation of spinal neuronal circuits that modulate the activity of another motoneuron pool (e.g., by reciprocal inhibition).

The simulation system was developed with the following requirements: flexibility to assemble and customize spinal cord circuitry, simulation of isolated or interconnected motor nuclei, replication of neurophysiological experiments and access via the Internet. The simulator control panels allow changing parameters of neuron and synaptic models, applying stimuli to the soma, dendrite or axon, visualizing results as graphs or exporting the raw data. The system is intended for studying individual neurons or complex neuronal circuitry, covering important aspects in the investigation of the human spinal cord motor control.

Another important feature is the simulation of the Hreflex, the homologue of the stretch reflex, obtained by electrical stimulation of peripheral nerves. H-reflex-based experiments have been largely used in research on the normal or pathologic spinal cord, in order to assess the excitability of the Ia afferent-motoneuron loop and to study the dynamics of interneuronal pathways through appropriate conditioning of the H-reflex (Misiaszek 2003; PierrotDeseilligny and Burke 2005). However, this technique is associated with many complexities (technical and in the interpretation of the results) that inhibit its broader use in 
clinical neurophysiology (Misiaszek 2003). The simulation of the H-reflex in different experimental paradigms can lead to useful interpretations of the experimental results obtained from humans.

The developed simulation system, named as ReMotoRemote Motoneuron Network Simulator, is a free software open source project, designed in a web-based architecture, being available for use at the site http://remoto.leb.usp.br. The source code can be downloaded at the same URL. A specific use of the simulator was published in a conference paper (Cisi and Kohn 2007).

\section{Methods}

The simulation system can be configured for studying any spinal cord motor nuclei. In its default configuration, the neuronal circuitry is related to motor nuclei involved with ankle flexion and extension. These nuclei are associated with the following muscles: soleus (SOL), medial gastrocnemius (MG), lateral gastrocnemius (LG) and tibialis anterior (TA). The total number of neurons simulated for these nuclei is shown in Table 1, based on estimates from human and cat literature (Feinstein et al. 1955; Cooper 1966; Ariano et al. 1973; Johnson et al. 1973; Buchthal and Schmalbruch 1980; Dum and Kennedy 1980; McComas 1991; Jankowska 1992; Carr et al. 1998; Banks 2006). Interneuron numbers are underestimated, in order to improve system performance. Users can change these numbers and also select how many nuclei will take part in a given simulation.

The numbers of neurons and axons in Table 1, adopted as default values in the simulator, are supported by experimental data, although may not exactly reflect the numbers found in human motor nuclei (mainly the interneurons). A few model parameters were adjusted (as reported later) so that these chosen default numbers of

Table 1 Default numbers of neurons and afferent fibers in each motor nucleus

\begin{tabular}{lcccc}
\hline & \multicolumn{2}{c}{ Extension } & & Flexion \\
\cline { 2 - 4 } & SOL & MG & LG & TA \\
\hline MN S & 800 & 250 & 200 & 250 \\
MN FR & 50 & 125 & 100 & 50 \\
MN FF & 50 & 125 & 100 & 50 \\
Ia afferent & 400 & 80 & 76 & 280 \\
Ib afferent & 200 & 40 & 38 & 140 \\
& & Extension & & Flexion \\
IN RC & & 350 & & 350 \\
IN IaIn & & 350 & & 350 \\
IN IbIn & & 350 & & 350 \\
\hline
\end{tabular}

neurons could give rise to behaviors similar to those found in human electrophysiological experiments.

Nevertheless, the user may change the numbers of elements, by interacting with the simulator directly on the Neural Pools panel in the Configuration module.

Simulated neurons are arranged as columns within the spinal cord, mimicking real neuron columns described in the cat (Scott and Mendell 1976; Burke et al. 1977; Burke 1981; Kernell 1986; Vanderhorst and Holstege 1997). The literature shows that: (1) the MG MN pool is coextensive with the SOL MN pool (Scott and Mendell 1976; Burke 1981); (2) the LG MN pool is situated slightly rostral to the MG MN pool, with a region of overlap (Scott and Mendell 1976); (3) FF type MNs have a tendency to be more rostral than S type MNs, whereas FR type MNs are more spread in a motor nucleus (Burke et al. 1977; Kernell 1986); (4) the MG nucleus has up to 300 MNs spread over 6 to $7 \mathrm{~mm}$ (Burke et al. 1977; Burke 1981), which leads to a density of $50 \mathrm{MN} / \mathrm{mm}$ in a spinal cord column.

Based on the cited experimental data and on the adopted numbers of neurons for humans (Table 1), two neuron columns were modeled: the first one containing SOL, MG and LG neurons and the second one containing TA neurons, arranged as follows:

1. For each nucleus, MNs are placed equally distributed in a line, ordered by their sizes $(\mathrm{S}<\mathrm{FR}<\mathrm{FF})$, from caudal to rostral positions.

2. SOL MNs are placed from an arbitrary caudal position (origin of the first column) to a rostral position, extending over $18.0 \mathrm{~mm}$.

3. MG MNs are placed from the same origin, extending over $10.0 \mathrm{~mm}$, overlapping with the first part of the SOL pool.

4. LG MNs are placed from position 10.0 to $18.0 \mathrm{~mm}$, overlapping with the last part of the SOL pool.

5. TA MNs are placed in a column apart, with another arbitrary origin, extending over $7.5 \mathrm{~mm}$.

6. A group of RCs, IaIn and IbIn interneurons is equally distributed among MNs of the extensor nuclei. Other group of such interneurons is equally distributed among MNs of the flexor nucleus.

The spatial positioning of the neurons in the simulated neuronal columns is specially important because RC synapses on MNs (and vice-versa) occur among synergistic nuclei (McCurdy and Hamm 1994a, b), with synaptic strength depending on the distance between the cells (Cullheim and Kellerth 1978; Windhorst 1996; Burke 2004).

During configuration, the user can alter the origin and end of each motor nucleus. In the same way, graphical interfaces can be used to select appropriate stimuli (both endogenous and exogenous) to be applied to the neuronal 
circuitry. The stimuli can be the descending drive from the brain, current injected inside the neurons or electrical stimulation on the simulated nerves. Users can assemble networks by means of a connectivity table and adjust neuronal or synaptic parameters in order to investigate normal or pathologic conditions, mimicking real, hypothesized or idealized situations.

Figure 1 shows a general view of the simulated motor nuclei receiving connections from the descending tracts (upper panel), a view of two antagonistic motor nuclei in the so-called reciprocal inhibition interconnection (middle panel) and details of the inner structure of one motor nucleus (lower panel).

The motor nuclei receive synaptic inputs from a few independent pathways: Ia and Ib sensory afferents, corticospinal and rubrospinal tracts, besides the connections among the nuclei neurons. The descending tracts generate spikes configured with one of two possible interspike interval distributions. Group Ia and Ib sensory axons can generate spikes in response to the nerve stimulation (with starting and stopping times selected by the user).

Two nerves are included in the simulator: the posterior tibial nerve (PTN) and the common peroneal nerve (CPN). The former contains axons from the SOL, MG and LG motor nuclei, and the latter contains axons from the TA nucleus. Electrical stimulation can be applied to these nerves (Fig. 1, bottom) at selected stimulation frequencies, amplitudes and starting times, simulating orthodromic and antidromic axonal activation as used in reflex experiments (e.g., H-reflex technique and reciprocal inhibition between agonist and antagonist nuclei).

\subsection{Software details}

The simulation environment may be divided into three modules: configuration, execution and analyses. The configuration module, with a set of default values, is a data entry for the models' parameters. The user can choose and configure a simulation scenario for running experiments and change properties of motoneurons, interneurons, neural tracts, motor-units, synaptic conductances and stimuli. It is possible to include or exclude neurons from each nucleus and save customizations in the server for future use. Figure 2 shows a configuration window of the simulator.

The execution module, which is the core of the system, solves differential equations of motoneurons and interneurons using a fourth order Runge-Kutta method, with default simulation step size of $0.05 \mathrm{~ms}$. Neurons, synaptic conductances and all other simulation elements are designed in an object-oriented architecture, implemented in Java classes. Simulations are run on parallel threads in order to improve software performance.
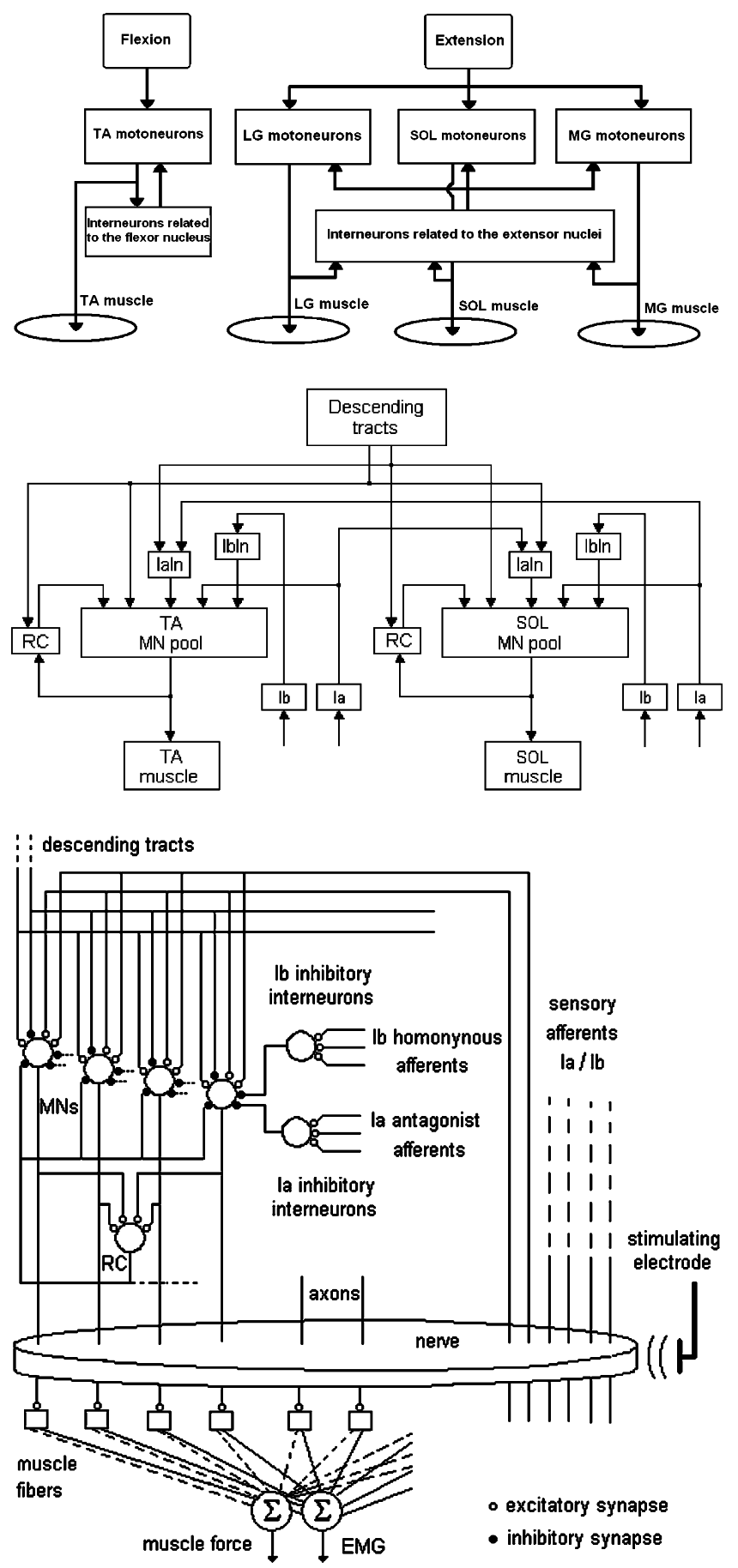

Fig. 1 Top General view of the modeled spinal cord motor nuclei. Middle Descending drive actuating on two antagonistic motor nuclei in order to control the SOL and TA muscles at the ankle joint. Below Structure of a spinal cord motor nucleus, its sensory afferents, and the associated nerve and muscle

The analyses module shows the results of a simulation, with the following options: occurrence times of spikes from each neuron, membrane potential of neurons, muscle force and EMG from individual or grouped motor-units, firing rate of the neurons, interspike interval histogram and a summary of results from all simulated neurons. For more 


\section{Home \\ Electrical stimulation \\ Tutorial \\ Bibliography \\ User \\ Scenario \\ Simulation \\ Configuration \\ Neural pools \\ Synaptic drive \\ Electrical stimulation \\ Injected current \\ Pool Soleus \\ Synapses \\ Motoneurons \\ Interneurons \\ Sensory fibers \\ Motor-units \\ $\mathrm{V}$-d conductances \\ Neuron \\ compartment \\ Basal current \\ Modulation superimposed on the basal current \\ Miscellaneous \\ $\Gamma \mathrm{S}$ \\ $\ulcorner\mathrm{FR}$ \\ $\ulcorner\mathrm{FF}$

\begin{tabular}{|l|}
\hline soma \\
\hline soma \\
\hline soma
\end{tabular} \\ amp [nA] \\ 0.0 \\ 0.0 \\ 0.0 \\ Results \\ Graphics \\ Files \\ Summary}

Fig. 2 Configuration window of the electrical stimulation parameters

refined signal processing or statistical analyses, the user can save files in ASCII format, with firing times, force, EMG, membrane potential data or conductance time course.

The simulation system development adhered to the ModelView-Control software architecture, where tasks are divided into layers of common responsibilities. Data access objects, running on the model layer, use JDBC library to save information in the application database. Java Server Pages (JSP), HTML and JavaScript are used in the view layer, which is responsible for the user interface. Action servlets, using Struts framework, are deployed in the control layer, where resides the business logic. All information about neurons, synapses and stimuli are stored in the database and loaded according to the simulation scenario selected by the user.

Open source software was used in order to reduce costs and take advantage of well-structured libraries. The main applications and libraries used in the system development are the Tomcat servlet container (http://tomcat.apache.org), Struts web framework (http://struts.apache.org), Eclipse Java IDE (http://eclipse.org), HSQLDB database manager system (http://hsqldb.org) and the JFreeChart chart library (http://www.jfree.org).

\subsection{Models of neurons and synapses}

One of the principles behind the modeling of the elements of the simulator was to keep a balance between biological realism and computational load. Therefore, the neuron models were chosen with a maximum of two compartments. The number of ionic channels was kept to a minimum that still enabled the reproduction of a reasonably large set of neuronal properties described in the literature. All the neuronal parameters (except those for the axons) were based on cat data and are assumed to be similar for humans (Jankowska and Hammar 2002).

\subsubsection{Motoneuron and interneuron models}

Motoneurons were designed as a compartmental integrateand-fire model, along with a functional structure to emulate the axon firing. The two compartments represent the soma and the dendritic tree, with electrotonic properties and modeling aspects based on Fleshman et al. (1988) and Rall et al. (1992). The soma and dendritic compartments were adopted with cylindrical geometry.

At the soma, a membrane capacitance $\left(C_{\mathrm{s}}\right)$ is in parallel association with leakage $\left(g_{\mathrm{ls}}\right)$, potassium $\left(g_{\mathrm{Ks}}\right.$ and $\left.g_{\mathrm{Kf}}\right)$ and sodium $\left(g_{\mathrm{Na}}\right)$ conductances, responsible for the suprathreshold electrical behavior. The dendritic compartment contains a leakage conductance $\left(g_{\text {ld }}\right)$ along with a membrane capacitance $\left(C_{\mathrm{d}}\right)$. A coupling conductance $\left(g_{\mathrm{c}}\right)$ links the two compartments. Synapses are modelled by conductances placed on both compartments, as will be described later. Injected current can be applied in both compartments for test purposes. Figure 3 shows an equivalent electric circuit of the motoneuron model. 
The axonal structure is functionally described in a software algorithm that generates spikes in response to suprathreshold electrical stimulation, applied at any point along its length. Somatic or axonal spikes reach their target with delays proportional to the conduction velocities and the covered distances.

Interneurons were modeled in a simpler manner, due to the relative scarcity of published data, having all conductances placed in one compartment, and no need of coupling conductance. Nevertheless, the mathematical modeling follows the same formalism as for motoneurons.

In the simulator all membrane potentials have values with respect to the resting membrane voltage. This means that if a neuron is in a resting state, its membrane voltage will be zero. Accordingly, Nernst equilibrium potentials will have values such as $+120 \mathrm{mV}$ for sodium and $-10 \mathrm{mV}$ for potassium.

Equations (1), (2), (3), (4), (5), (6), (7), and (8) describe the membrane potentials of the dendrite and soma compartments of the motoneuron models.

$$
\begin{aligned}
& C_{\mathrm{d}} \frac{\mathrm{d} V_{\mathrm{d}}(t)}{\mathrm{d} t}=-I_{\mathrm{syn}-\mathrm{d}}(t)-g_{\mathrm{ld}}\left(V_{\mathrm{d}}(t)-E_{\mathrm{l}}\right) \\
& -g_{\mathrm{c}}\left(V_{\mathrm{d}}(t)-V_{\mathrm{s}}(t)\right)+I_{\mathrm{inj}-\mathrm{d}}(t) \\
& C_{\mathrm{s}} \frac{\mathrm{d} V_{\mathrm{s}}(t)}{\mathrm{d} t}=-I_{\mathrm{syn}-\mathrm{s}}(t)-g_{\mathrm{ls}}\left(V_{\mathrm{s}}(t)-E_{\mathrm{l}}\right) \\
& -g_{\mathrm{c}}\left(V_{\mathrm{s}}(t)-V_{\mathrm{d}}(t)\right)-I_{\text {ion }}(t)+I_{\text {inj-s }}(t) \\
& I_{\text {ion }}(t)=\bar{g}_{\mathrm{Na}} m^{3} h\left(V_{\mathrm{s}}(t)-E_{\mathrm{Na}}\right)+\bar{g}_{\mathrm{Kf}} n^{4}\left(V_{\mathrm{s}}(t)-E_{\mathrm{K}}\right) \\
& +\bar{g}_{\mathrm{Ks}} q^{2}\left(V_{\mathrm{s}}(t)-E_{\mathrm{K}}\right) \\
& g_{\mathrm{c}}=\frac{2}{\frac{R_{\mathrm{i}} \cdot l_{\mathrm{d}}}{\pi \cdot r_{\mathrm{d}}^{2}}+\frac{R_{i} \cdot l_{\mathrm{s}}}{\pi \cdot r_{\mathrm{s}}^{2}}} \\
& g_{\mathrm{ld}}=\frac{2 \pi \cdot r_{\mathrm{d}} \cdot l_{\mathrm{d}}}{R_{\mathrm{m}-\mathrm{d}}} \\
& g_{\mathrm{ls}}=\frac{2 \pi \cdot r_{\mathrm{s}} \cdot l_{\mathrm{s}}}{R_{\mathrm{m}-\mathrm{s}}} \\
& C_{\mathrm{d}}=2 \pi \cdot r_{\mathrm{d}} \cdot l_{\mathrm{d}} \cdot C_{\mathrm{m}} \\
& C_{\mathrm{s}}=2 \pi \cdot r_{\mathrm{s}} \cdot l_{\mathrm{s}} \cdot C_{\mathrm{m}}
\end{aligned}
$$

In these equations, $V_{\mathrm{d}}(t)$ and $V_{\mathrm{s}}(t)$ are the dendritic and somatic membrane potential; $E_{1}$ is the leak equilibrium potential; $g_{1 d}$ and $g_{\text {ls }}$ are the leak conductance for dendrite and soma; $g_{\mathrm{c}}$ is the coupling conductance; $C_{\mathrm{d}}$ and $C_{\mathrm{s}}$ are
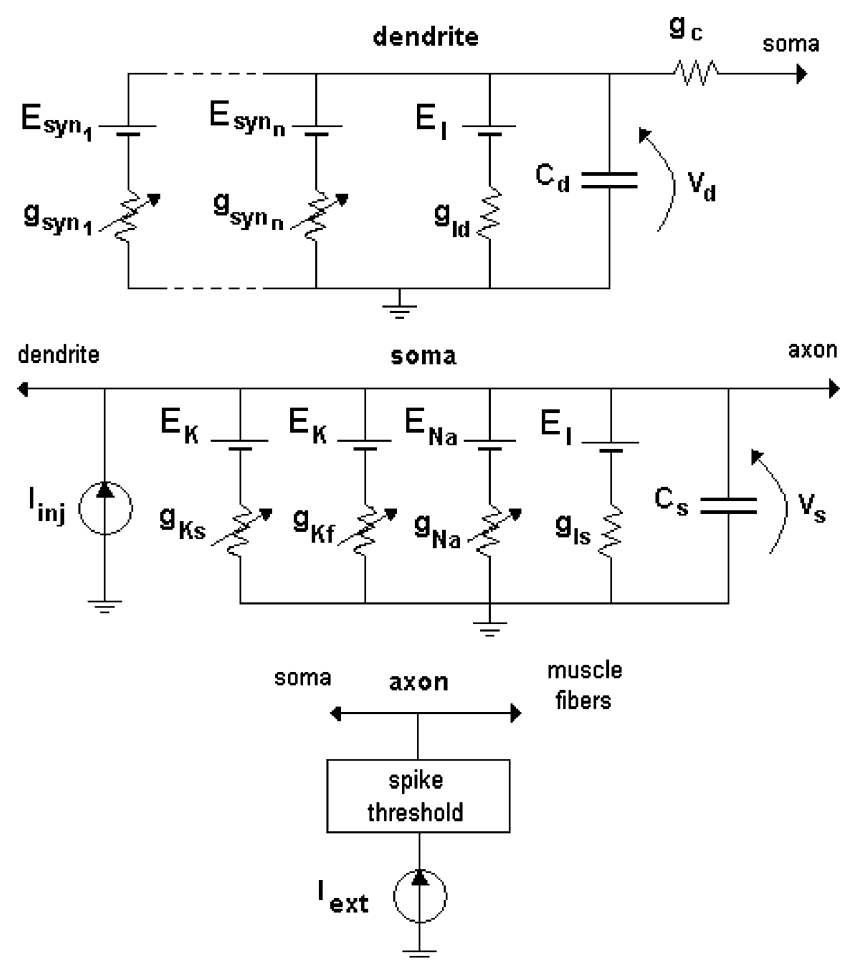

Fig. 3 Equivalent electric circuit of the motoneuron model. Synapses and injected current can be attached to both compartments (soma or dendrite). They are represented only in one compartment to simplify the figure. The resting levels are adopted as $0 \mathrm{mV}$. $I_{\mathrm{ext}}$ is the external current source, applied by skin electrodes

dendritic and somatic membrane capacitances; $C_{\mathrm{m}}$ is the membrane specific capacitance; $R_{\mathrm{i}}$ is the cytoplasm resistivity; $R_{\mathrm{m}-\mathrm{d}}$ and $R_{\mathrm{m}-\mathrm{s}}$ are dendritic and somatic membrane specific resistances; $l_{\mathrm{d}}, l_{\mathrm{s}}, r_{\mathrm{d}}$ and $r_{\mathrm{s}}$ are the dendritic and somatic compartment length and radius; $I_{\text {syn-d }}(t)$ and $I_{\text {syn-s }}(t)$ are the postsynaptic currents caused by independent synaptic connections on the dendritic or somatic compartment (further described); $I_{\mathrm{inj}-\mathrm{d}}(t)$ and $I_{\mathrm{inj-s}}(t)$ are the injected currents, inside the dendrite and the soma, for test purposes; $I_{\text {ion }}(t)$ is the membrane current due to the voltage-dependent ionic conductances $\left(g_{\mathrm{Na}}, g_{\mathrm{Kf}}\right.$ and $\left.g_{\mathrm{Ks}}\right) ; m, h, n$ and $q$ are state variables, whose time evolution depends on voltagedependent rates $\left(\alpha_{m}, \beta_{m}, \alpha_{h}, \beta_{h}, \alpha_{n}, \beta_{n}, \alpha_{q}\right.$ and $\left.\beta_{q}\right) ; \bar{g}_{\mathrm{Na}}$, $\bar{g}_{\mathrm{Kf}}$ and $\bar{g}_{\mathrm{Ks}}$ are the maximal conductances of the sodium, fast potassium and slow potassium currents, with equilibrium potentials of $E_{\mathrm{Na}}=120 \mathrm{mV}$ and $E_{\mathrm{K}}=-10 \mathrm{mV}$. The leakage Nernst voltage was $E_{1}=0 \mathrm{mV}$.

The voltage-dependent rate constants were modeled according to the pulse-based model (Destexhe 1997), which simplifies the Hodgkin and Huxley formulation. The afterhyperpolarization (AHP), an important feature that regulates neuron firing rates, was incorporated in the neuron models by adding a slow potassium conductance. The combined approaches are briefly described in what follows. 
The differential equations in $m(t), h(t), n(t)$ and $q(t)$, each in the form $\dot{\gamma}=\alpha_{\gamma} \cdot(1-\gamma)-\beta_{\gamma} \cdot \gamma$, can be solved analytically if the time courses of the rates $\left(\alpha_{m}, \beta_{m}, \alpha_{h}\right.$, $\beta_{h}, \alpha_{n}, \beta_{n}, \alpha_{q}$ and $\beta_{q}$ ) are approximated by rectangular pulses, triggered when the membrane potential crosses a given threshold (Destexhe 1997). When the threshold is crossed, the rectangular pulses, assumed to last $0.6 \mathrm{~ms}$, will be going from 0 to peak values equal to $\alpha_{M}, \alpha_{N}, \beta_{H}, \alpha_{Q}$ for the variables $\alpha_{m}, \alpha_{n}, \beta_{h}, \alpha_{q}$, respectively. On the other hand the rectangular pulses ( $0.6 \mathrm{~ms}$ duration) will be going from constant values equal to $\alpha_{H}, \beta_{M}, \beta_{N}, \beta_{Q}$ towards zero when the threshold is crossed, for variables $\alpha_{h}, \beta_{m}, \beta_{n}, \beta_{q}$, respectively. This will cause exponential variations in the state variables $m(t), h(t), n(t)$ and $q(t)$ before, during and after the pulses, with time constants depending on the respective alphas and betas (Destexhe 1997).

The maximal values of the conductances $\left(\bar{g}_{\mathrm{Na}}, \bar{g}_{\mathrm{Kf}}\right.$ and $\left.\bar{g}_{\mathrm{Ks}}\right)$ and the pulse amplitudes of the rates were determined from published experimental data. Their values were chosen to match experimental motoneuron parameters or functions, such as the AHP (Zengel et al. 1985) and $f \times I$ relation (for step injected current; Kernell 1965; Schwindt and Crill 1984; Binder et al. 1996) and may be found online at the simulator menu, under V-d conductances. For example, the default values of the rate parameters associated with the AHP are $\alpha_{Q}=1.5 \mathrm{~ms}^{-1}$ and $\beta_{Q}=0.025 \mathrm{~ms}^{-1}$. This means that the conductance that causes the AHP will be an exponentially increasing function until time $0.6 \mathrm{~ms}$, when it becomes an exponentially decreasing function. The inverses of the values $\alpha_{Q}$ and $\beta_{Q}$ multiplied by two [because one has $q^{2}$ in Eq. (3)] define the rising and falling time constants of the conductance responsible for the AHP.

As the MNs in the simulator can be stimulated by current injection in the soma and as rheobase measurements from different types of MNs are available in the cat literature, the rheobase is selectable in the configuration window "Motoneurons" (the default rheobase values used in the simulator are given in Table 2). Before running a simulation, the user may view the mean values of several parameters (for S, FR and FF types) by selecting "see properties" in the "Motoneurons" window. This includes the values of the resultant mean voltage thresholds, which the system computed from Ohm's law (by multiplying the rheobase and the somatic input resistance). After running a simulation, the user may view some data that include the input resistance and the voltage threshold of each MN, by selecting "Summary" in the Section 3 (at the bottom left of any window), or selecting "Files"-"Properties-All MNs".

MN parameters in a pool were attributed considering the ordering from the smallest to the largest $\mathrm{MN}$, following the size-order $(\mathrm{S}<\mathrm{FR}<\mathrm{FF})$. Each $\mathrm{MN}$ is indicated by an index which represents its size-rank, MN S1 indicating the smallest $\mathrm{S}$ type $\mathrm{MN}, \mathrm{MN} \mathrm{S} 2$ the next one and so on. Range of values for electrotonic parameters are shown in Table 2. The default electrotonic properties adopted are based on the "step model" (Fleshman et al. 1988; Rall et al. 1992). Membrane specific capacitance was set to $1.0 \mu \mathrm{F} /$ $\mathrm{cm}^{2}$ and cytoplasm resistivity was set to $70.0 \Omega . \mathrm{cm}$ for all neurons (Barret and Crill 1974; Fleshman et al. 1988).

The value of a MN parameter was set after a linear interpolation of the given range, considering the MN type and index. For example, for a simulation of a single S-type $\mathrm{MN}$, the axon threshold would be equal to $18.0 \mathrm{~mA}$. For a simulation with 3 S-type MNs, the axon thresholds would be 18.0, 15.2 and $12.4 \mathrm{~mA}$ for $\mathrm{MN} \mathrm{S} 1, \mathrm{~S} 2$ and $\mathrm{S} 3$, respectively. A similar rule would apply to FR and FF MNs, within their respective ranges of parameter values. Some of the parameter ranges in Table 2 contribute to the size-ordered recruitment pattern among MNs activated by a common input (Henneman et al. 1965).

Interneuron electrotonic properties were adopted according to data published by Bui et al. (2003), but adjusted to a one-compartment interneuron model and with the added assumption that $\mathrm{Ib}$ interneurons are similar to Ia interneurons. For Renshaw cells, these values were adapted so that the membrane time constant could make the cell fire a burst of action potentials in response to a supra-threshold input (Hultborn and Pierrot-Deseilligny 1979). The values of

Table 2 Range of values (minimum-maximum) for the modeled motoneuron parameters

\begin{tabular}{lccc}
\hline Parameter & S MN & FR MN & FF MN \\
\hline Rheobase current $(\mathrm{nA})$ & $3.5-6.5$ & $6.5-17.5$ & $17.5-25.1$ \\
Soma diameter $(\mu \mathrm{m})$ & $77.5-82.5$ & $82.5-87.5$ & $87.5-113$ \\
Soma length $(\mu \mathrm{m})$ & $77.5-82.5$ & $82.5-87.5$ & $87.5-113$ \\
Soma specific resistance $\left(\mathrm{k} \Omega . \mathrm{cm}^{2}\right)$ & $1.15-1.05$ & $1.05-0.95$ & $0.95-0.65$ \\
Dendrite diameter $(\mu \mathrm{m})$ & $41.5-62.5$ & $62.5-83.5$ & $8.8-8.1$ \\
Dendrite length $(\mathrm{mm})$ & $5.5-6.8$ & $10.7-6.95$ & $8.1-10.6$ \\
Dendrite specific resistance $\left(\mathrm{k} \Omega . \mathrm{cm}^{2}\right)$ & $14.4-10.7$ & $12.4-12.2$ & $6.95-6.05$ \\
Axon threshold $(\mathrm{mA})$ & $18.0-12.4$ & $47.0-50.0$ & $12.2-12.0$ \\
Axon conduction velocity $(\mathrm{m} / \mathrm{s})$ & $44.0-47.0$ & $50.0-53.0$ \\
\hline
\end{tabular}

Motoneurons of all motor nuclei have the same range of values, by default 
parameters such as rate constants and peak conductances (for default values, see V-d conductances panel in the Configuration module of the simulator) were determined so that the AHP, the $f \times I$ relation and the generation of action potential bursts followed data from the literature (Hultborn and Pierrot-Deseilligny 1979; Cleveland et al. 1981; Walmsley and Tracey 1981; Windhorst 1990; Windhorst 1996; Uchiyama et al. 2003). Parameters related to the IaIn and IbIn interneuron conductances were chosen so that these interneurons would fire isolated action potentials in response to a volley coming from the sensory afferents (Jankowska 1992). The default values may be found in the "V-d conductances" window of the simulator, after selecting the desired interneuron in the Pool and Neuron pulldown menus.

Motor axons $\mathrm{MN}$ axons have the default length of $0.8 \mathrm{~m}$, a typical value for distal leg muscles for an adult human. Distance between the stimulation point and the spinal cord is set by default to $0.6 \mathrm{~m}$ for the PTN nerve, and $0.66 \mathrm{~m}$ for the CPN nerve. Distance between the stimulation point and the end-plate is set by default to $0.2 \mathrm{~m}$ for the PTN nerve, and $0.14 \mathrm{~m}$ for the CPN nerve. These values were estimated in the laboratory. The user can alter all these lengths and distances, for simulation of primate, cat or other animals.

Orthodromic and antidromic volleys can be elicited in response to suprathreshold electrical stimulation of motor axons. The antidromic action potential may collide with an orthodromic spike and cause annihilation. If there is no down-going action potential from the motoneuron soma, the antidromic action potential excites the Renshaw cells (Fig. 1, bottom) and discharges and action potential in the soma after a conduction delay.

\subsubsection{Neuronal dynamics}

Starting a simulation, somatic and dendritic membrane potentials are at their resting levels, set to $0 \mathrm{mV}$. Excitatory synapses or injected current can make the soma membrane of a given neuron surpass the firing threshold. In response, sodium conductance rises quickly, depolarizes the soma and hence generates an action potential. The potassium conductances are also activated: the fast potassium conductance repolarizes the membrane after the spike, whereas the slow potassium conductance leads the membrane to the AHP period.

A new action potential is fired when the soma membrane potential reaches the firing threshold and the absolute refractory period is over. Another possibility for a soma spike generation is the arrival of an antidromic spike coming from the axon, assuming no refractoriness is under way from a previous activation. The default value of the
$\mathrm{MN}$ absolute refractory period in the simulator was set at $5.0 \mathrm{~ms}$, to keep the maximum firing rate at 200 spikes $/ \mathrm{s}$ (Powers 1993). For brevity, spikes/s is indicated as pps, pulses $/ \mathrm{s}$, in the simulator. The axon may trigger an action potential if the external stimulation overcomes the axonal firing threshold.

\subsubsection{Synapse modeling}

Synaptic actions are effected by changes in a conductance placed at a postsynaptic neuron compartment (dendrite or soma). These conductances are activated in response to action potentials generated by presynaptic neurons. The membrane current caused by independent synaptic connections is defined by the following equation:

$I_{\mathrm{syn}}(t)=\sum_{i=1}^{N} \sum_{j=1}^{N} g_{\mathrm{syn} i j}(t)\left(E_{\mathrm{syn} i j}-V(t)\right) \quad i \neq j$

where $g_{\text {syn } i j}(t)$ is the time course of an independent synaptic conductance, connecting the presynaptic neuron $i$ with the postsynaptic neuron $j ; E_{\mathrm{syn}} i j$ is the reversal potential related to the synapse type $(70 \mathrm{mV}$ for excitatory synapses and $-16 \mathrm{mV}$ for inhibitory synapses); and $V(t)$ is the membrane potential of the compartment where the synapse is located (dendritic or somatic compartment). For cells not interconnected, $g_{\text {syn } i j}(t)$ is null.

The synaptic conductance behavior is controlled by equations that model the release, binding and unbinding of neurotransmitters, modeled by the two state Markov model proposed by Destexhe et al. (1994a). In this proposal, the transmitter-gated postsynaptic conductance is given by $g_{\text {syn }}(t)=g_{\max } \cdot r(t)$, where $r(t)$ takes values in the range $[0,1]$ and $g_{\max }$ is the maximum conductance of the synapse. The variable $r(t)$, representing the fraction of bound postsynaptic receptors with respect to the total amount of receptors, is a rising exponential function during the release of neurotransmitter (mimicked by a rectangular pulse of neurotransmitter, with peak value $T_{\max }$ ), and a decreasing exponential function after the neurotransmission. This method of computing synaptic conductance is preferable to using alpha functions (Rall 1967), because the results are more precise and response saturation occurs naturally, as the conductance value approaches its upper limit (Destexhe et al. 1994b; Giugliano 2000). Moreover, the conductance computation of the Markov model can be optimized by using Lytton's algorithm (Lytton 1996), which assembles the actions of all synapses in four groups: those for which transmission is in course and those for which transmission is over, for excitatory and inhibitory synapses. 
Excitatory synapse parameter values were based on Finkel and Redman (1983) and inhibitory synapse (except for RCs) on Stuart and Redman (1990). Synaptic conductances of RCs to MNs had their maximum value based on Hultborn et al. (1988) and Friedman et al. (1981). Synaptic conductances of MNs to RCs had maximum conductance values chosen to cause bursts in the RCs (Walmsley and Tracey 1981; Windhorst 1996).

The synapses may be configured to have special recovery dynamics of releasable neurotransmitter stores in order to mimic synaptic depression. Whenever a presynaptic neuron discharges an action potential, the available amount $s(t)$ of stored (and releasable) neurotransmitter diminishes by a fraction $p$ and returns exponentially to its resting level with a time constant $\tau$ (Kohn et al. 1995; Abbott et al. 1997). A lower amount of released neurotransmitter decreases the peak value of the postsynaptic conductance when activated by a presynaptic action potential and hence will cause a smaller postsynaptic potential. This feature of synaptic depression is found in the Ia to MN synapses (Capek and Esplin 1977) and in the $\mathrm{MN}$ to RC synapses (Hultborn and Pierrot-Deseilligny 1979). In the simulation system, the default values of the fraction $p$ and time constant $\tau$ were based on data from the papers cited above and also on data evidencing the dynamics of human $\mathrm{H}$ reflex depression (Floeter and Kohn 1997). This means that the values of $p$ and $\tau$ for the synapses between Ia terminals and the motoneurons were adjusted so that the simulator could reproduce at the network level reflex amplitude decreases similar to those described for humans. The adopted values for $p$ and $\tau$ are 0.11 and $1,500 \mathrm{~ms}$ for the synapses of Ia axons on motoneurons (also used in Cisi and Kohn 2007), and 0.50 and $200 \mathrm{~ms}$ for the synapses between the motoneurons and the Renshaw cells.

Recurrent inhibition synapses The influence of RCs on the $\mathrm{MN}$ pool is widespread and a single RC reaches several MNs (McCurdy and Hamm 1994a; Windhorst 1996). On the other hand, one MN reaches a smaller number of RCs (Cullheim and Kellerth 1978; Burke 2004). The influences of a $\mathrm{RC}$ over the $\mathrm{MN}$ pool and the influence of a $\mathrm{MN}$ over the RC pool diminish with distance, modeled according to Eq. (10):

weight $=\frac{a}{a+d^{2}}$

where weight is the synaptic strength, which multiples the conductance value of a synapse between a $\mathrm{RC}$ and a $\mathrm{MN} ; d$ is the distance between a $\mathrm{RC}$ and a $\mathrm{MN}$ in the rostro-caudal axis; $a=0.22$ is a decay factor, chosen to achieve $10 \%$ of the maximum strength at a distance of $1.4 \mathrm{~mm}$ between a RC and target MNs (McCurdy and Hamm 1994a; Windhorst 1996). For the synapses from MNs to RCs, the factor $a$ was set at 0.01 , because the maximum spread of the MN collaterals reaches $1 \mathrm{~mm}$ (Cullheim and Kellerth 1978; Burke 2004).

Synaptic noise Synaptic noise was also considered in the system. Vertebrate neurons receive a very large number of active synaptic inputs that result in a random membrane potential. This effect was modeled by using synaptic conductances activated by Poisson processes, which represent spike times of a population of pre-synaptic neurons. The system allows application of independent excitatory and/or inhibitory synaptic noise over each simulated MN, sharing the same formalism used for the synapses between two cells.

\subsubsection{Motor-unit force and action potentials}

The motor-unit twitch in response to an action potential in the associated motoneuron was modeled as the impulse response of a critically damped second-order system [Eq. (11)]; Fuglevand et al. 1993). The motor-unit spike train is represented in Eq. (12). Equation (13) gives the force generated by one $\mathrm{MU}$, under isometric conditions.

$$
\begin{aligned}
& a(t)=A_{\text {peak }} \frac{t}{t_{\text {peak }}} \exp \left(1-\frac{t}{t_{\text {peak }}}\right) u(t) \quad t \in \Re \\
& e(t)=\sum_{i} \delta\left(t-t_{\mathrm{AP}_{i}}\right)
\end{aligned}
$$

$f(t)=e(t) * a(t)$

where $a(t)$ is the function that models one twitch; $A_{\text {peak }}$ and $t_{\text {peak }}$ are the peak and time-to-peak (or contraction time) of the twitch; $u($.$) is the Heaviside step function; f(t)$ is the force developed by one $\mathrm{MU} ; e(t)$ is the system input, i.e., the superposition of all MU action potentials occurring at times $t_{\mathrm{AP} i}$.

To improve the simulation performance, a twitch was viewed as the impulse response of a linear time-invariant system. The discrete-time version of force twitch was obtained, with great computational advantage, by discretizing the critically damped second-order differential equation by the "impulse invariance" digital filter technique (Oppenheim et al. 1999). Hence, one can simulate the twitch generation by a difference equation. 
Table 3 Twitch, force and MUAP parameter ranges

\begin{tabular}{lccc}
\hline Parameter & S MU & FR MU & FF MU \\
\hline Twitch force (gram-force) & $10.5-12.5$ & $12.5-30.0$ & $30.0-50.0$ \\
Tetanic force (gram-force) & $40.0-50.0$ & $50.0-120.0$ & $120.0-200.0$ \\
Contraction time (ms) & $110-100$ & $73.5-55.5$ & $82.3-56.9$ \\
$A_{M}$ parameter $(\mu \mathrm{V})$ & $0.105-0.125$ & $0.125-0.30$ & $0.30-0.50$ \\
$\lambda_{M}$ parameter $(\mathrm{ms})$ & $0.80-0.70$ & $0.70-0.60$ & $0.60-0.50$ \\
\hline
\end{tabular}

The impulse response is sampled at every $T$ time units, where $T$ is the numerical integration time step [Eq. (14)]. The times of motor unit activation are indicated by $n_{i}$ [Eq. (15)]. The $z$-transform yields the desired difference equation, obtained in Eq. (16), which computes the force developed by one motor-unit. When the motor unit is activated by a fast train, there will be an increase in motor unit force, due to summation of twitches, until a preestablished maximum value is reached, the tetanic force (see Table 3). In the present implementation, for simplicity reasons, the saturation is hard, meaning that, at any time point, a force value above the selected tetanic force will saturate. The sum of forces from all motor-units of a muscle represents the muscle force [Eq. (17)]. The equations in discrete-time are:

$a(n)=T \cdot A_{\text {peak }} \frac{T \cdot n}{t_{\text {peak }}} \exp \left(1-\frac{T \cdot n}{t_{\text {peak }}}\right) \cdot u(n) \quad n \in \mathrm{Z}$

$e(n)=\sum_{i} \delta\left(n-n_{i}\right)$

$f(n)=2 e^{\frac{-T}{t_{\text {peak }}}} f(n-1)-\exp \left(\frac{-2 T}{t_{\text {peak }}}\right) f(n-2)$

$+\frac{A_{\text {peak }} T^{2}}{t_{\text {peak }}} \exp \left(1-\frac{T}{t_{\text {peak }}}\right) e(n-1)$

$F(n)=\sum_{i=1}^{N} f_{i}(n)$

where $a(n), e(n)$ and $f(n)$ are the sampled versions of Eqs. (11), (12) and (13); and $F(n)$ is the total muscle force.

The motor-unit action potentials (MUAPs) were modeled using Hermite-Rodriguez functions, which fit the shape of MUAPs well (Lo Conte et al. 1994; Zhou and Rymer 2004). Surface bipolar electrodes were assumed, with $8 \mathrm{~mm}$ diameters and $20 \mathrm{~mm}$ separation, as typically used in human experiments. They are usually placed in the skin in a middle position between the muscle center and a distal tendon.

The first-order Hermite-Rodriguez function [Eq. (18)] models biphasic MUAPs, whereas the second-order
Hermite-Rodriguez function [Eq. (19)] models triphasic MUAPs. A simulated motor-unit had the same probability to be associated with an $\mathrm{HR}_{1}$ function or an $\mathrm{HR}_{2}$ function.

$\mathrm{HR}_{1}(t)=A_{M}\left(t-t_{A P}\right) e^{-\left(\frac{\left(t-t_{A P}\right)}{\lambda_{M}}\right)^{2}} u\left(t-t_{A P}\right)$

$\mathrm{HR}_{2}(t)=A_{M}\left[1-2\left(\frac{\left(t-t_{A P}\right)}{\lambda_{M}}\right)^{2}\right] e^{-\left(\frac{\left(t-t_{A P}\right)}{\lambda_{M}}\right)^{2}} u\left(t-t_{A P}\right)$

where $A_{M}$ and $\lambda_{M}$ are scale and time factors and $t_{\mathrm{AP}}$ is the time of arrival of an action potential.

These two equations represent MUAPs at the center of a motor-unit territory. The position of the motor-unit territory in relation to the surface electrodes could alter the MUAP shape. Motor-unit territories were considered as spread in a random way within a muscle section (Johnson et al. 1973).

The surface electromyogram (EMG) is not a simple summation of all MUAPs generated by the motor-units. It is affected by at least three effects: (1) biological tissues filter the original signal, altering the frequency spectrum (Plonsey 1974); (2) MUAPs due to distant motor-units are more attenuated than those due to closer motor-units (Fuglevand et al. 1992); (3) in practice, the electromyogram is band-filtered in order to avoid noise and aliasing. All these three effects are represented in the simulator.

The MUAP amplitude attenuation is modeled to fit data shown by Fuglevand et al. (1992), and is represented by Eq. (20). The duration increase with distance between muscle fibers and the electrodes was based on data from Hermens et al. (1992), and is represented by Eq. (21).

$V=V_{0} \exp \left(\frac{-d}{\tau_{\text {at }}}\right)$

$T=T_{0}(1+C \cdot d)$

where $V$ is the surface MUAP amplitude, $V_{0}$ is the MUAP amplitude at the center of the motor-unit territory, $T$ is the 
surface MUAP duration, $T_{0}$ is the MUAP duration at the center of the motor-unit territory, $d$ is the distance between the center of the motor-unit territory and the middle point of the two electrodes; $\tau_{\text {at }}$ is the attenuation distance constant and $C$ is a constant related to the widening of the MUAP. $\tau_{\text {at }}$ was set at $5.0 \mathrm{~mm}^{-1}$ in order to fit to the modeled attenuation (Fuglevand et al. 1992), and $C$ was set at $0.1 \mathrm{~mm}^{-1}$ (Hermens et al. 1992). The human muscle sections were based on Maganaris et al. (1998), with diameters (in mm) set to $18.4,17.0,18.8$ and 18.8 for the SOL, MG, LG and TA, respectively.

The simulated myoelectric signal can also be filtered to mimic bandpass filtering typically employed in electromyography. The simulator contains a band-pass filter implemented as a digital filter, using the technique of "impulse invariance" (Oppenheim et al. 1999).

Motor-unit twitch properties were based on Garnett et al. (1979), Vandervoort and McComas (1983) and Chan et al. (2001). The relationship between twitch peak and the amplitude of the MUAP (at its origin) was adopted as linear, based on the fact that both have linear relations to the number of muscle fibers of a motor-unit. MUAP time factor $\left(\lambda_{M}\right)$ was chosen between 5 and $40 \mathrm{~ms}$ to match MUAP durations found in measurements taken in our laboratory and those shown in the literature (Fuglevand et al. 1992). Different ranges of parameter values were used for different motor-unit types and sizes, as can be seen in Table 3. However, data from human leg motor units are incomplete and scarce, therefore, some of the values are probably only rough approximations. These are the default values found in the simulator, but these values may be changed at will every time a user runs a new simulation.

\subsubsection{Electrically stimulated sensory afferents}

Group Ia and Ib sensory afferents have axons of large diameter. In spinal reflex studies, they are easily recruited in response to electrical stimulation, applied by surface electrodes. In the simulator, each sensory afferent axon may generate and transmit spikes through its axon in response to electrical external stimulation, applied to the nerves.

Table 4 shows sensory axon threshold ranges for nerve stimulation with a $1.0 \mathrm{~ms}$ electrical pulse (the only pulse duration included at present in the simulator), and axon conduction velocity ranges based on Jimenez et al. (1970) and Awiszus and Feistner (1993). Axon length and

Table 4 Sensory axon parameter ranges

\begin{tabular}{lrr}
\hline & Ia afferent & Ib afferent \\
\hline Axon conduction velocity (m/s) & $69.0-65.0$ & $66.0-62.0$ \\
Axon threshold (mA) & $6.0-18.0$ & $13.0-22.0$ \\
\hline
\end{tabular}

stimulation point are the same for sensory and motor axons because they are bundled together in the same nerve.

Sensory afferents in the simulator project to MN pools with a projection fraction that can be selected from 0 to $100 \%$, i.e., a single pre-synaptic axon can reach up to $100 \%$ of the MNs and INs in the target pool. For example, if a $90 \%$ connectivity is set for the synapses between soleus Ia afferent fibers and homonymous FF motoneurons, this means that, each Ia afferent will be synaptically connected to $90 \%$ of the FF type homonymous MNs, chosen in a random way. The random choices are independent from one Ia afferent to another. The (default) projection fraction depends on the connection type (homonymous or heteronymous), the motor nucleus and the neurons involved.

\subsubsection{Descending tracts}

Descending tracts that drive the motor nuclei were modeled as a set of axons, each being an independent spike train generator. It is possible to select and configure two different point process realizations for the spike train: Poisson or with truncated-Gaussian interspike intervals (ISI). The former mimics a very irregular spike train, for example due to the superposition of many independent point processes (Cox and Isham 1980). The latter may be set to a very regular spike train by selecting a small standard deviation for the interspike intervals.

The descending tract spike trains can be modulated, by varying the mean ISI according to a modulating signal: pulse, ramp, sinusoid or square wave. The same modulation can be applied to the injected currents.

As described above for the sensory fiber connections, descending tract fibers project to a given fraction (from 0 to $100 \%$ ) of a MN pool or a group of INs. Such fraction can be configured prior to a simulation.

The descending tracts in humans may activate the motoneuron pools either directly or through interneurons (Jankowska 1992), and the exact proportion is not well known for different motor nuclei in humans or animal experimental models. In the present simulator, the driving inputs from the upper centers are being called descending tracts either if their action is direct or indirect. What matters are the spike trains (e.g., Poisson) that activate the synapses on the motoneurons of each pool.

\subsection{Model tuning and validation}

As one of our purposes was to provide means for simulating human neurophysiology, some comments are due on the intricacies found in this process. First, neuronal characteristics used in the modeling, e.g., input resistance, membrane time constant, amplitude of post-synaptic potentials etc, cannot be obtained directly from human neurons, being 
taken from cat neurons instead. These data are probably reasonable first approximations to human data (Jankowska and Hammar 2002). Second, many different sources of data were used for the tuning and validation processes, which may cause a dispersion of parameter values due to different samples and techniques used in each laboratory. Third, some parameter values are not presently available from animal data, and hence had to be chosen ad-hoc. These issues are common to all papers that study human brain and spinal cord dynamics by means of computer simulations.

The model adjustment/validation was divided in two stages: initially, a set of fundamental properties of motoneurons and interneurons was adjusted, avoiding divergence in one property when another was being matched. Whenever possible, biophysically-based parameter values were used. After that, the circuitry as a whole was adjusted in order to exhibit global behaviors similar to those found in human experimental studies. For this second stage, measurements of force and EMG, distribution of motor-unit interspike intervals (ISI) and H-reflex results were used, as presented in other sections.

Motoneuron parameters were mainly based on data from cat motoneurons. The electrotonic parameters of the MN model (Table 2) were chosen with the intention of exhibiting the same membrane electrical properties as measured by Zengel et al. (1985). The properties shown by the models are well compliant with the corresponding properties of the referenced MNs. After the model adjustment process, simulated motoneurons exhibited the properties shown in Table 5, in columns 3-5. The second column in this table indicates the range of values found in the literature. The fit between the measurements from the simulated motoneurons and the experimental literature is a consequence of the chosen values of the MN electrotonic parameters (Table 2) and conductance parameters, being, therefore, a (partial) validation of the motoneuron models.

Having matched membrane electrical properties, the resulting relations between the steady-state discharge rate and the magnitude of the injected current ( $f \times I$ relations) also showed acceptable values.
For interneurons, the approach was similar, except that the available amount of experimental data is much less (see Section 2.2.1). In the case of the Renshaw cells the tendency for bursting was taken into account together with the available biophysical data. On the contrary, for Ia and Ib interneurons their tendency for single discharges was a goal for parameter adjustment.

\section{Results}

\subsection{Membrane potential time course}

Typical simulated neuron membrane potential time-courses can be visualized in the result module of the simulation system. Figure 4 shows the action potentials generated by an $\mathrm{S}$ type $\mathrm{MN}$ being activated by a corticospinal descending tract (100 axons), each fiber discharging either at a $200 \mathrm{pps}$ mean rate (above) or at 20 pps (below), all being independent Poisson trains. In the second simulation, the motoneuron membrane did not reach firing threshold, making it easier to notice the small excitatory postsynaptic potentials occurring randomly.

Membrane potential time-course of Renshaw cells, IaIn and IbIn interneurons can also be visualized in the Results module of the simulator. Figure 5 shows the membrane potential of a RC in response to a strong supra-threshold stimulus to the PTN nerve (all motor axons fired an action potential). The RC, as happens in the cat (Walmsley and Tracey 1981), fired a burst of action potentials (in this simulation there were ten spikes followed later by two additional spikes).

\subsection{Firing rate adaptation}

Figure 6 shows the firing rate adaptation of representative S, FR and FF type motoneurons submitted to current steps of injected current. Firing rate adaptation was observed for all motoneuron types, practically ending after the first or second interspike interval. In cat motoneurons, the basal

Table 5 Properties exhibited by the modeled motoneurons

\begin{tabular}{|c|c|c|c|c|}
\hline & \multirow{2}{*}{$\frac{\text { Real MNs }}{\text { S-FR-FF }}$} & \multicolumn{3}{|c|}{ Modeled MNs } \\
\hline & & $\mathrm{S}$ & FR & $\mathrm{FF}$ \\
\hline Input resistance $(\mathrm{M} \Omega)$ & $1.6-0.9-0.6$ & 1.6 & 0.9 & 0.6 \\
\hline Membrane time constant (ms) & $10.4-8.0-5.9$ & 10.4 & 8.0 & 5.9 \\
\hline AHP magnitude $(\mathrm{mV})$ & $4.9-4.3-3.0$ & 4.9 & 4.3 & 3.0 \\
\hline AHP duration $(\mathrm{ms})$ & $161-78-65$ & 160 & 87 & 67 \\
\hline$f \times I$ steady state first segment $(\mathrm{pps} / \mathrm{nA})$ & 1 to 3 & 2.7 & 2.5 & 3.6 \\
\hline$F \times I$ steady state second segment $(\mathrm{pps} / \mathrm{nA})$ & 3 to 8 & 6.3 & 3.8 & 4.9 \\
\hline
\end{tabular}

For the $f \times I$ relations, experimental data were taken from Schwindt and Crill (1984); for the other properties, data were taken from Zengel et al. (1985) 

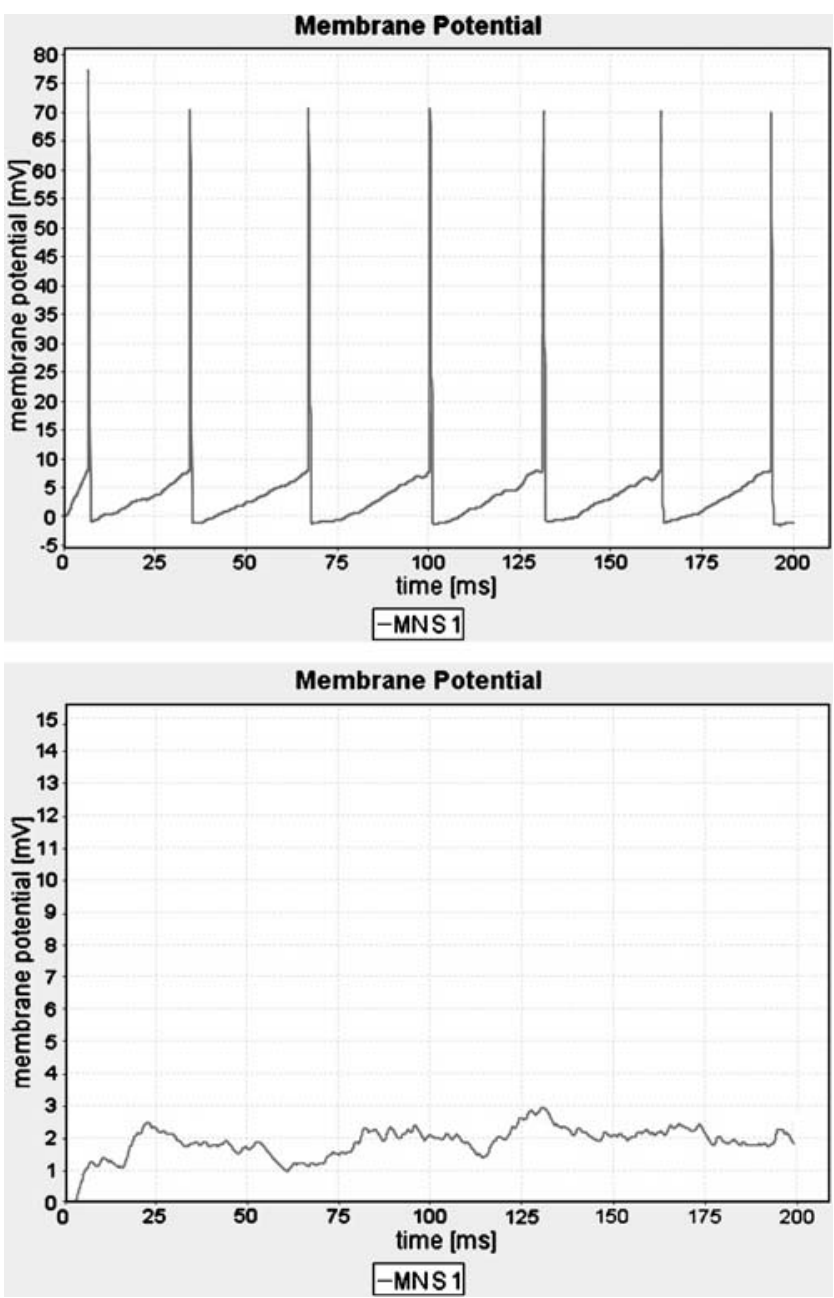

Fig. $4 \mathrm{MN}$ membrane potential time-course. Top $\mathrm{MN}$ receiving spikes from 100 independent descending axons discharging at 200 pps. Bottom Same as above, but with descending axons discharging at $20 \mathrm{pps}$. The two panels were reproduced directly from the graphical output of the simulator

firing rate is usually approached within the first few interspike intervals, being followed by other phases of adaptation which are not represented in our models (Binder et al. 1996).

\subsection{Response of motoneurons to linearly increasing descending drive}

In this simulation, the aim was to observe the firing rate of a few $\mathrm{S}$ type motor units from a pool when subjected to a linearly increasing intensity of the descending drive. The muscle simulated is the tibialis anterior, with $250 \mathrm{~S}$ type MNs, 50 FR type and $50 \mathrm{FF}$ type. The descending tract was simulated with 70 axons, with a $100 \%$ connectivity, and each with a basal Poisson process with mean interspike interval of $20 \mathrm{~ms}$ superimposed on a linear increase in intensity with peak 50 spikes/s. Synaptic noise (imple-

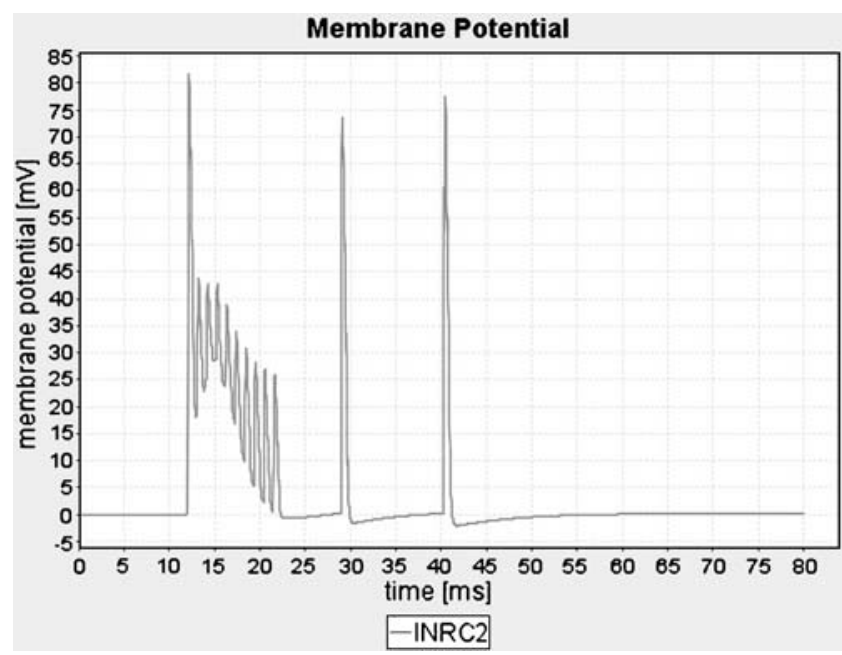

Fig. 5 Renshaw cell membrane potential time-course in response to supra-threshold stimulation to the PTN nerve

mented as independent Poisson processes applied to each $\mathrm{MN}$, as described in Section 2) had a $10 \mathrm{~ms}$ mean ISI.

Figure 7 shows the mean firing rates (smoothed by a three-point sliding average) of MNs 1, 25, 50, 75 and 100 (all type S) from the pool. The firing rate of a smaller MN tended to be higher than that of a larger MN. The total muscle force is drawn in thick line and shows a nearly monotonic increase. Its level at $1,000 \mathrm{~ms}$ was about $50 \%$ the maximum voluntary contraction (MVC) as checked with another simulation where a high intensity descending drive was employed that recruited the whole pool at full rate. In this type of simulation there can be local changes in the order of recruitment due to random synaptic noise used in the simulations. These results are reasonably compatible with the experimental results found by De Luca et al. (1996) in human tibialis anterior muscle (see their Fig. 4).

(a)

(b)
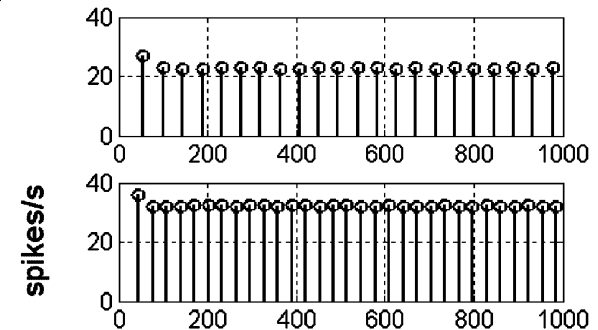

(c)

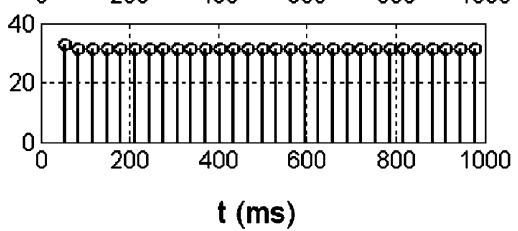

Fig. 6 Firing rate adaptation of motoneurons of types S (a), FR (b) and $\mathrm{FF}$ (c) after injection of current steps in their somas. The ordinate indicates the instantaneous firing rate associated with each interspike interval. The figure was done in Matlab from the spike rate files generated by the simulator 


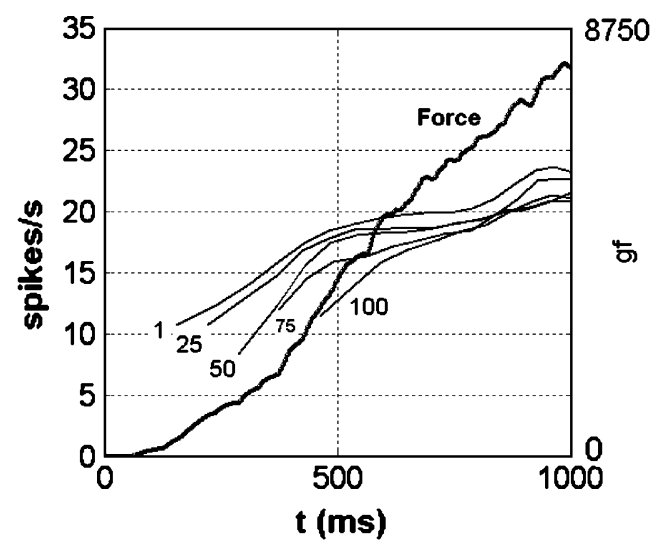

Fig. 7 Mean firing rates of five type $S$ motoneurons from a simulation of the tibialis anterior motoneuron pool. The descending drive spike trains had a linearly increasing intensity. The thick line indicates the resulting muscle force with the ordinate indicated on the right side

\subsection{Force generation and MN recruitment}

A computer-simulated single motor unit twitch had a timeto-peak, or contraction time, equal to that set in the MotorUnits Parameters configuration panel. For example, for a pool containing only $100 \mathrm{~S}$ type $\mathrm{MNs}$, with a range of timeto-peak from 100 to $110 \mathrm{~ms}$ (from larger to smaller MUs) the time-to-peak of MN 50 was $105 \mathrm{~ms}$. On the other hand, the half-relaxation time (the interval from the peak of the twitch to half the peak value) resulted approximately $170 \mathrm{~ms}$. This value seems larger than the (scarce) available data from human leg motor units (Garnett et al. 1979; Vandervoort and McComas 1983; Andreassen and ArendtNielsen 1987). The slow decay of the impulse response of the second order system that generates the twitches may perhaps be adequate for the smallest human soleus motor units, but not for other human muscles.

The default parameter values of twitch amplitudes and tetanic forces resulted in physiologically reasonable slopes of the force $\mathrm{x}$ frequency $(F \times f)$ functions for single motor units. For simulations of a type $\mathrm{S} M N$ subjected to trains of pulse stimuli at rates from $2 \mathrm{~Hz}$ up to $40 \mathrm{~Hz}$, the resulting $F \times f$ plot was a straight line (saturating at $40 \mathrm{gf}$ ) with a slope of $7.9 \% / \mathrm{Hz}$, which is within the range found in human toe extensors (Macefield et al. 1996) and cat medial gastrocnemius muscle (Kernell et al. 1983). The shape of the $F \times f$ relation did not follow the sigmoidal shape found in real motor units because of the simple approach used in the simulator (see Section 4).

Spinal cord motoneuron pools receive a multitude of synaptic inputs from different parts of the central and peripheral nervous systems. The time course of the firing patterns of the motoneurons will define the dynamic behavior of the innervated muscle. To evaluate the dynamics behind force generation in the model, the following simulation was carried out, mimicking a twitch interpolation experiment. A voluntary contraction was induced with the activation of the excitatory corticospinal tract, composed of 200 axons, each one discharging independently as a Poisson process. The intensity of the Poisson processes varied linearly from 10 up to $60 \mathrm{pps}$, the latter being reached after $300 \mathrm{~ms}$ and maintained at this value from then on. This produced a rising activity in the motoneuron pool as shown in Fig. 8. Both recruitment of new units (following the size-ordered recruitment) and increase in firing rate are visible in the figure. An electrical stimulus was applied at $1,000 \mathrm{~ms}$ to the posterior tibial nerve with an intensity appropriate to discharge the largest motor units. For simplicity, here we are assuming that an external stimulus activates preferentially the largest diameter axons, which is observed in animal setups (but not necessarily in human experiments). The response of the motoneuron pool may be seen in Fig. 8 by the discharge of a large number of the higher threshold motoneurons. The corresponding force recordings associated with such a simulation are shown in Fig. 9. In Fig. 9(a) one sees the twitch caused by a single electrical stimulus to the PTN nerve applied at 1,000 ms, under the assumption of relaxed muscle, i.e., without any descending drive being applied to the motoneuron pool. When the descending drive was applied (generating the motoneuron pool discharges of Fig. 8) the MG muscle generated the force shown in Fig. 9(b). The force gradually rose to a steady state and then at $1,000 \mathrm{~ms}$ there was a superimposed twitch due to the electrical stimulation.

For a comparison with experimentally obtained data, a simple experiment was run in the lab (with local ethics committee approval) mimicking the idea behind the simulation described above. A subject (male, age 36), sitting comfortably, had its right foot strapped to a pedaltorque-meter system. In the first experiment the subject sat

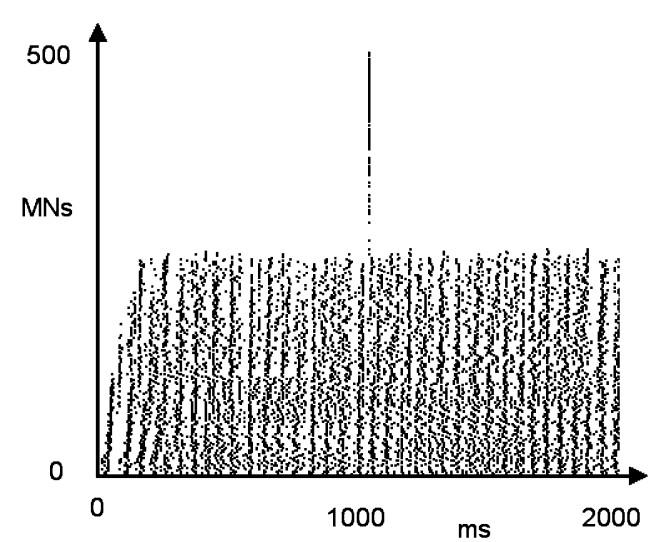

Fig. 8 Raster plot of motoneuron firings from the MG muscle from a simulation of a ramp-and-hold descending activation of the motoneuron pool. At 1,000 ms there was a strong electrical stimulus to the posterior tibial nerve which discharge preferentially the largest motor units 

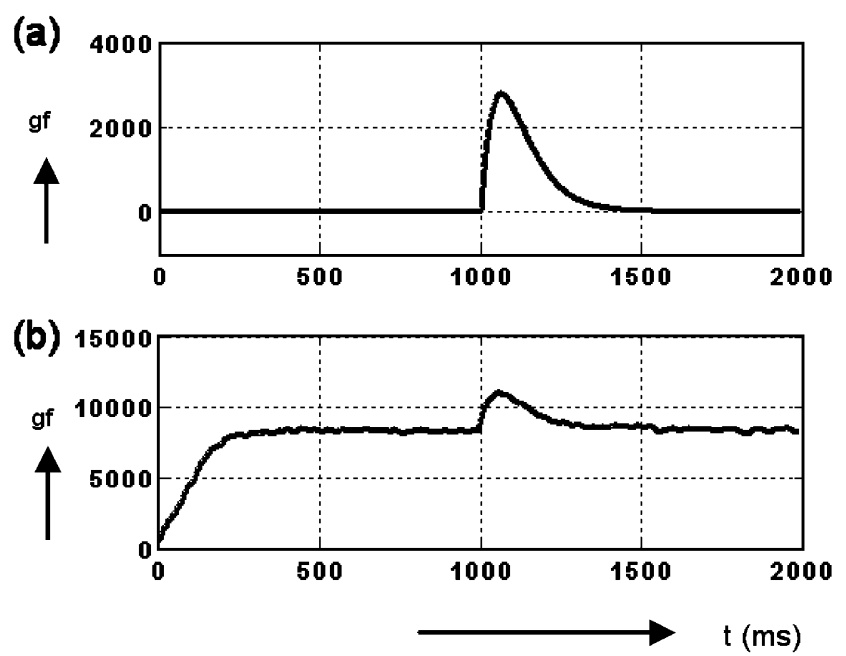

Fig. 9 Force developed by the MG muscle in a simulation of a single electrical stimulus applied to the posterior tibial nerve with the muscle relaxed (a), or while reaching a plateau torque (b). This last situation (b) corresponds to the raster plot shown in Fig. 8

relaxed and a single electrical pulse stimulus $(100 \mu \mathrm{s})$ was applied at the popliteal fossa. The stimulus, in this subject, activated only the medial gastrocnemius muscle, without any reflex activity. The resulting torque twitch is shown in Fig. 10(a). Then, the subject was instructed to apply a raising force up to a predefined plateau level (low torque value) while the same electrical stimulus was applied at a latency of 1,000 ms. The resulting torque is shown in Fig. 10(b) showing a rise towards a plateau (the subject was not able to keep a constant level) and then a superimposed twitch due to the electrical stimulation.

Note that the ordinate calibrations are different in Figs. 9 and 10 , the former being in force and the latter in torque. But the general behaviors are similar, which suggests that the simulator may provide useful predictions of results from neurophysiological experiments. What the simulation gives in addition to the experimentally obtainable data are the firing times of all motoneurons in the pool (Fig. 8). With present technology, one could record the firing times of only a very small (random) sample of the existing motor units from a human muscle such as the MG.

Force modulation A rhythmic voluntary contraction of a given muscle, common in everyday life, must be achieved by modulating the descending drive intensity in a rhythmic fashion. In order to illustrate the force production in response to a time-varying descending command, the $\mathrm{MG}$ motor nucleus was subjected to a sinusoidally modulated descending drive intensity. This signal affected the mean firing rate of each corticospinal fiber composing the descending command. The simulated motoneurons had also connections with associated RCs. In this simulation, the corticospinal tract was formed by 100 fibers, whose firing rate varied between 100 and $500 \mathrm{pps}$, modulated by a $1 \mathrm{~Hz}$ sinusoid signal. As a result, the force developed by the MG muscle presented a sinusoidal aspect, containing some level of distortion. Figure 11 shows, from top to bottom, the spike times of all fibers of the corticospinal tract (first $2 \mathrm{~s}$ ), the MG force produced and the spike times of all MG MNs. There are no equivalent data from humans for comparison. The force and the firing times of a few motor units could be recorded from an experimental subject, but the whole picture is not obtainable.

\subsection{Motor-unit ISI histogram}

Experimental measures of motor-unit ISI can be obtained from humans with special procedures (Clamann 1969; Andreassen and Rosenfalck 1980; Rosenfalck and Andreassen 1980; Halonen et al. 1981). Different motor-unit ISI distributions have been found, according to the muscle and the contraction level (Clamann 1969; Person and Kudina 1972; De Luca and Forrest 1973; Poliakov et al. 1995). In some cases, the distribution is Gaussian-like (usually for faster discharge rates), whereas in others, the distribution is asymmetric, with a prolonged right tail (usually for slower firing rates). For example, Andreassen and Rosenfalck (1980) have found the mean ISI for their sample of TA motor-units (from 71 motor units in four subjects) ranging from 49 to $160 \mathrm{~ms}$, and with the corresponding ISI standard deviation varying from about 6 to $28 \mathrm{~ms}$. They mention the ISI histograms were nearly normal, with a slight skew to longer intervals.

A simulation was performed to analyze the statistics of the ISI of S type TA motor-units. The TA motor nucleus
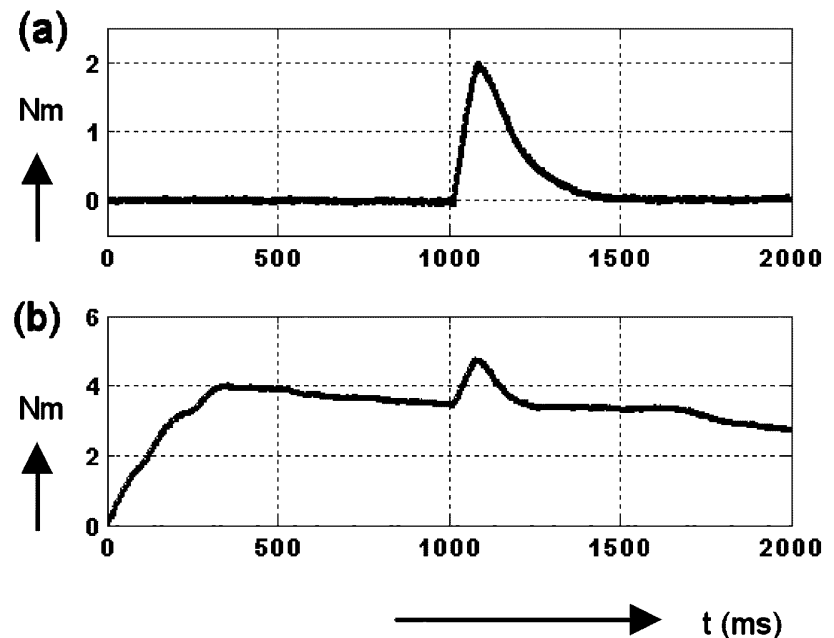

Fig. 10 Force developed by the MG muscle in a human subject in response to a single electrical stimulus applied to the posterior tibial nerve with the muscle relaxed (a), or while trying to keep a constant torque (b) 


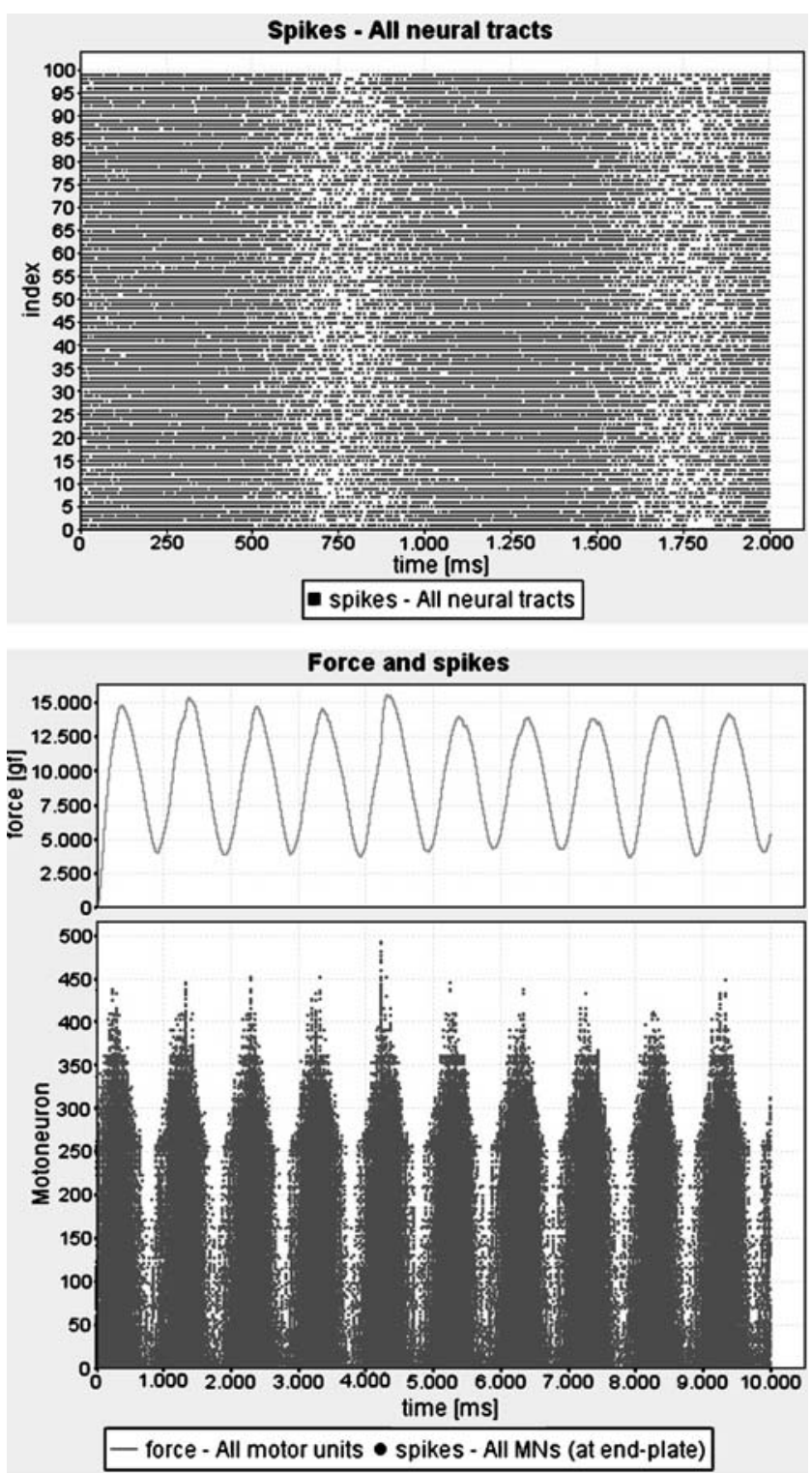

Fig. 11 To $p$ First $2 \mathrm{~s}$ of the sinusoidally modulated spike times of each corticospinal fiber. Middle Force generated by the MG muscle during $10 \mathrm{~s}$. Bottom The corresponding $\mathrm{MN}$ pool spike times (ordinate are the $\mathrm{MN}$ indexes, from smaller to larger)

was subjected to drive from the corticospinal tract (100 axons) discharging as independent Poisson trains with mean rates 300 spikes/s. The first type $\mathrm{S} \mathrm{MN}$ of the pool (MN 1) had a Gaussian-like (as judged visually) ISI histogram [Fig. 12(a)], with coefficient of skewness 0.232. The corresponding mean interval was $53.79 \mathrm{~ms}$, the standard deviation was $4.63 \mathrm{~ms}$ and, therefore, the $\mathrm{CV}$ was 0.086. A larger type S MN from the pool, MN 91, had a more asymmetric ISI histogram, with a coefficient of skewness 1.201 [Fig. 12(b)]. The mean interval was $75.27 \mathrm{~ms}$, the standard deviation was $15.41 \mathrm{~ms}$ and the $\mathrm{CV}$ was 0.205 . This finding of lower $\mathrm{CV}$ of the ISIs for a faster discharging motoneuron was also found if the same MN was simulated with two different descending drive intensities (values not shown). The pairs of values of mean interval and standard deviation determined from the simulations of the MG motoneuron pool are compatible with the experimental data presented by Andreassen and Rosenfalck (1980) in their Fig. 3.

\subsection{H-reflex experiment}

H-reflexes can be obtained from many muscles, but for lower limb experiments the soleus muscle is the most commonly assessed. The soleus H-reflex is obtained by placing a stimulation electrode over the PTN nerve at the popliteal fossa. The volley of action potentials from the activated Ia sensory fibers reaches the spinal cord and activates excitatory synapses on the motoneuron pool. The firing of a fraction of the MN pool generates an efferent volley, which causes a contraction of the innervated muscle. The reflex response is observed by recording the evoked electrical signal on the soleus muscle.

Experiments conducted in our laboratory, as well as those published in the literature (Floeter and Kohn 1997; Kohn et al. 1997; Pierrot-Deseilligny and Burke 2005), supplied data used to adjust the threshold for axon firing and the size of motor-unit potentials (MUAP). These parameters were changed in an ad-hoc way (within physiological ranges) until the simulated motor nuclei could generate H-reflexes compatible with those obtained in humans.

Figure 13 shows the soleus EMG signal and the corresponding motor-units that fired after applying a stimulation pulse to the PTN nerve, at a position equivalent to the popliteal fossa of the virtual subject. The stimulation pulse had amplitude of $14.0 \mathrm{~mA}$. The $\mathrm{M}$ wave arose with
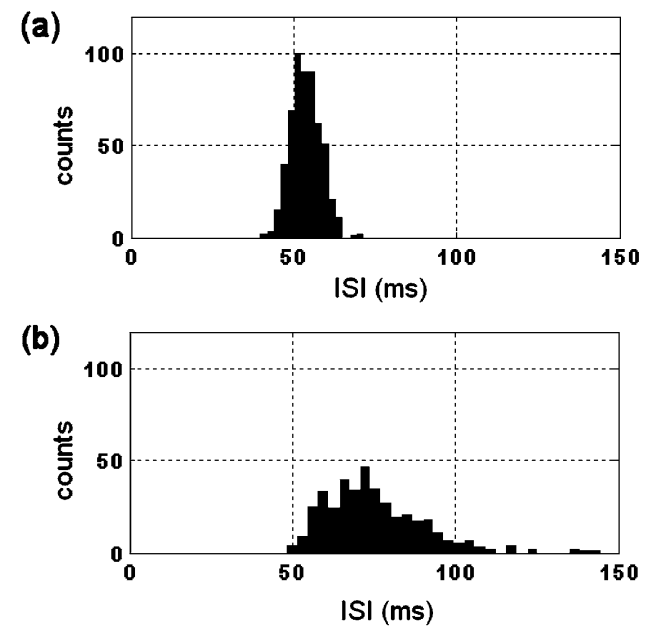

Fig. 12 ISI histograms of two $\mathrm{S}$ type motor-units from a simulated pool of motoneurons of the TA muscle. The histogram for MN 1 (a) resulted narrower and Gaussian-like, while that for MN 91 (b) resulted skewed to the right and wider 
latency around $5 \mathrm{~ms}$, as shown by the EMG signal and by the graph of MN spikes.

The graph of the MN spikes shows the times when the spikes reached the neuromuscular junction. As the distances covered by the spikes directly discharged by the motor axons are small, these spikes reach the neuromuscular junction early ( $\mathrm{M}$ wave at $\sim 5 \mathrm{~ms}$ ). Spikes associated with the H-reflex cover greater distances and reach the neuromuscular junction later ( $\mathrm{H}$ responses at $\sim 29 \mathrm{~ms}$ ). Although not visible in the figure, there are no double discharges in any of the MNs in the pool due to the refractory periods of the axons and soma. This can be checked by selecting "Files" and then "generate" in the "Results" option (at the bottom left) and then looking at the spike times of each MN. Notice that the MN spike times in the simulator may be shown or saved in a file in two different ways: with respect to the arrival at the end-plate (as used in Fig. 13) or with respect to the generation time at the soma (less preferable for comparisons with human electrophysiological data).

Figure 14 shows the H-reflex and $\mathrm{M}$ wave recruitment curves obtained from a simulation. These curves were obtained by applying increasing amplitude pulses over the simulated PTN and measuring the soleus peak-to-peak $\mathrm{H}$ reflex and $\mathrm{M}$ wave responses. These recruitment curves are within a range found experimentally (Floeter and Kohn 1997; Pierrot-Deseilligny and Burke 2005) and unpublished data supplied by M. K. Floeter and A. F. Kohn.

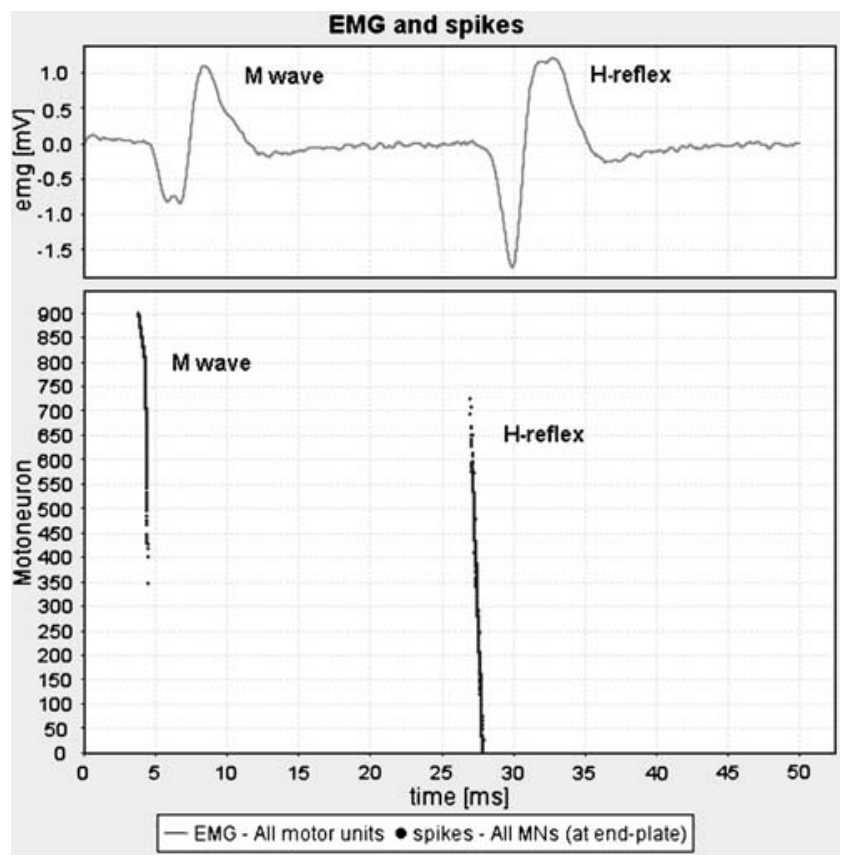

Fig. 13 Upper panel Soleus $M$ wave and H-reflex in response to a $14 \mathrm{~mA}$ pulse of stimulation. Lower panel Corresponding times of the MN spikes reaching the neuromuscular junction (MN indexes on ordinate)

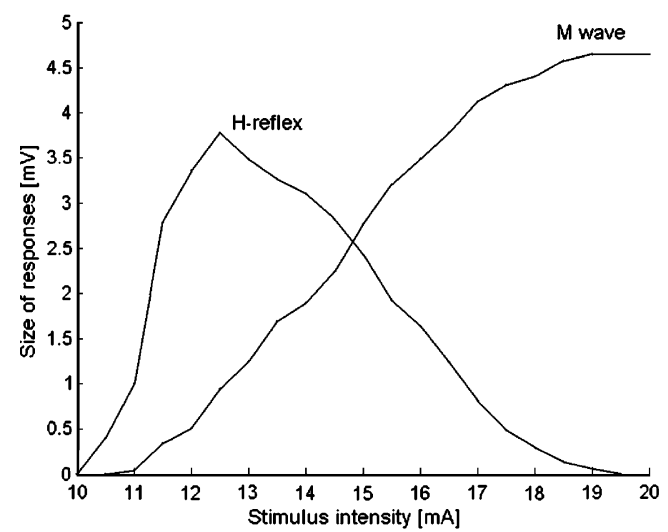

Fig. 14 H-reflex and $M$ wave recruitment curves, obtained in response to stimulation pulses with amplitude ranging from 10 to $20 \mathrm{~mA}$

H-reflex depression When H-reflexes are elicited in humans in response to a train of stimuli, e.g., electrical pulses delivered at $1 \mathrm{~s}$ intervals, there is a progressive decrease in H-reflex amplitude until a plateau value is reached after a few stimuli. This is the so called H-reflex depression, and has been attributed to the Ia-MN synaptic depression (Kohn et al. 1997), which reduces the number of reflexively recruited MNs.

An experimental H-reflex protocol (Floeter and Kohn 1997) was simulated consisting of the application of a $1 \mathrm{~Hz}$ train of ten pulses with constant intensity, over the PTN nerve, and verifying the decline of the peak-to-peak amplitudes of the H-reflexes. The synaptic depression parameters were kept the same for every synapse between the Ia afferents and all the MNs in the simulated motor nuclei. Results are shown in the panels of Fig. 15.

The upper panel of Fig. 15 shows the amplitude decline of the H-reflex until a plateau is reached. The lower panel of Fig. 15 shows the number of recruited MNs in response to each stimulation pulse. The depression of H-reflex amplitude obtained in the simulations is within the range found experimentally (Floeter and Kohn 1997; Kohn et al. 1997; Meunier et al. 2007). It is important to note that the data supplied in the lower panel are impossible to obtain in humans and cats with present technologies. Thus, this, as well as other simulations, can provide predictions about underlying mechanisms and phenomena that are otherwise unavailable.

\subsection{Performance}

A large simulation, as that of the H-reflex depression during $10 \mathrm{~s}$, lasted $8.9 \mathrm{~min}$, run on a system server based on two Xeon $3.0 \mathrm{GHz}$ dual core (64 bits) CPUs. Another simulation, in which all neurons of all nuclei were simulated during $1 \mathrm{~s}$, subjected to descending drive and 

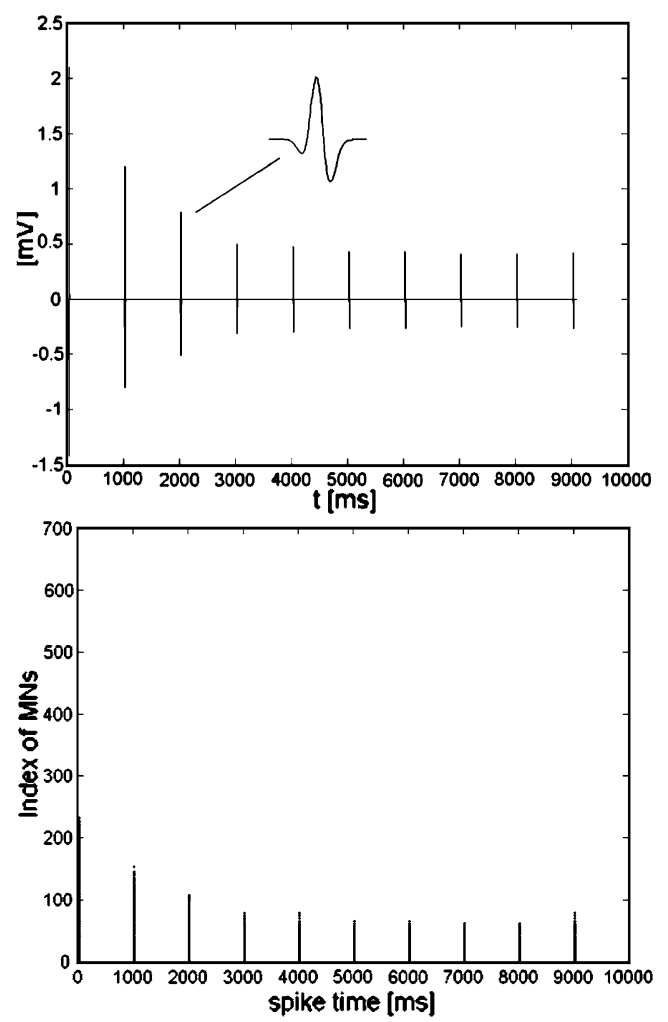

Fig. 15 Top H-reflexes evoked at every $1 \mathrm{~s}$, the peak-to-peak amplitude decreasing to a depression plateau. Inset Detail of H-reflex waveform from the simulation. Bottom Index of recruited MNs in response to each stimulation pulse

nerve electrical stimulation, lasted $13.1 \mathrm{~min}$. This simulation involved computations associated with 6,054 neurons and about 2,400,000 synapses.

\section{Discussion}

A first and much reduced version of the introduced simulation system was developed as a desktop application (Cisi and Kohn 2004), implemented in $\mathrm{C}++$ language. The expanded present version was developed as a Java application, running on an Internet server. In the past, scientific computation was restricted to languages such as Fortran and $\mathrm{C} / \mathrm{C}++$. Nevertheless, Java is proving its feasibility in high performance computation since technical consortiums have made improvements in the Java Virtual Machine. At present, programs written in Java can compete with programs written in other technical languages, being superior in some aspects as operating system portability, open-source library availability and appropriateness for offering the system as an Internet application.

The model tuning and validation tried to assure agreement with experimental results at the single neuron level, as well as with results emerging from the collective behavior of the spinal cord circuitry and the associated muscles and nerves. For this purpose, the validation process considered physiological characteristics of individual neurons, such as input-output dynamics, frequency versus current relations, firing rate adaptation and others, as well as collective characteristics, such as the "size principle" MN recruitment, H-reflex parameters and behavior, isometric force generation and EMG signal of single motor-unit and whole muscle. Overall, the most critical parameters to be adjusted were those associated with the voltagedependent conductances.

At the cellular level, most parameter values relied on measurements obtained from cats, due to the absence of human data. Even so, the circuitry parameters were adjusted to yield good quality reproductions of human electrophysiological data. The individual neuron models were chosen with either one or two compartments due to the computational costs of large network simulations. Many features of real motoneurons were appropriately reproduced by the developed models. The firing rate adaptation of the models, the so called initial or early-phase adaptation (Kernell 1972), resulted very short, dying out practically after the first or second interspike interval. However, motoneuron firing rate adaptation has more complex dynamics (Kernell and Monster 1982; Powers et al. 1999) than that represented in the simulator and improvements to the proposed models could increase the models' realism.

Due to the importance of persistent inward currents originating in the motoneuron dendrites (Heckman et al. 2005), an interesting incremental evolution of the present simulator would be the addition of an L-type calcium channel in the dendritic compartment (Booth and Rinzel 1995). Hopefully, the resulting increase in computation time would be tolerable. However, the neuronal models in the simulator could be improved in a wider sense to achieve an increased realism with the availability of a more powerful machine (e.g., a cluster) and the use of massively parallel algorithms (Hines et al. 2008). For example, the models could include (1) more types of ionic channels in the soma (e.g., calcium dependent potassium channels, calcium channels), (2) multiple dendritic compartments to represent the spatial complexity of the dendritic tree, and (3) dendritic voltage-dependent ionic channels (Dai et al. 2002; Taylor and Enoka 2004; Bui et al. 2006; Vieira and Kohn 2007). These would increase the repertoire of emergent properties obtainable from the simulator at the neuronal and network levels.

The default numbers of motoneurons in the simulator follow data from human literature (see Section 2). The spatial distribution of the motoneurons followed that described in cats, i.e., longitudinally spread along the spinal cord. This was similar to that adopted by Maltenfort et al. (1998) but different from Bashor (1998) and Subramanian et al. (2005) who adopted a distribution following a square 
grid, with wraparound at the four edges. While the latter choice may be advantageous in terms of visualization when all motoneurons have uniform properties, it could be more cumbersome when the motoneurons are modelled with different dynamics, according to S, FR and FF types.

To keep the computational demand within reasonable levels, the default numbers of interneurons were adopted smaller than those estimated in cats (they are not known in humans). The network behavior was not affected since the corresponding synaptic parameters were adjusted to compensate for the reduced numbers of interneurons.

At the neuronal network level, several properties arise naturally in the simulator. For example, in an entirely deterministic situation, the minimum discharge rate of the motoneurons in the simulated pool increases with motoneuron size (not shown in Section 3). This was found to be an important issue in the experimental and simulation studies of Moritz et al. (2005) of the relations between muscle force variability and mean muscle force. They employed the model of Fuglevand et al. (1993) that imposes the motoneuron relations algorithmically.

Other studies in the literature have dealt with the synchronization between motor-units and its effects on muscle force (Datta et al. 1991; Yao et al. 2000; Mochizuki et al. 2005). In the present simulator, motor unit synchronization may be obtained naturally (not algorithmically) by choosing appropriately the descending drive connectivity so that a sufficient amount of input divergence guarantees the required level of motor unit synchronization. The simulator thus provides a tool for testing the effects of different patterns of descending inputs on a motoneuronal pool and also on force generation.

The simulations of the H-reflex and other electrophysiological protocols, such as those involving reflex-conditioning by antagonist Ia and homonymous $\mathrm{Ib}$ electrical activation (Pierrot-Deseilligny and Burke 2005), are capable of providing several measurements not available from the non-invasive human experiments. For example, it is possible to characterize all motoneurons' discharge patterns in different experimental situations, which are not known experimentally. Additionally, the qualitative and quantitative effects of inhibition of the motoneuron pool caused by Ia and $\mathrm{Ib}$ interneurons (as discharged by external stimuli to a nerve) can be analyzed.

Simulation of the dynamics of spinal cord motor nuclei should be helpful in the interpretation of the experimental results from humans and in the planning of new experiments. The simulator has been used to create hypothesis on spinal mechanisms (Cisi and Kohn 2007) and also as a teaching aid in a graduate course. On the other hand, theoretical neuroscientists will have a tool to test their theoretical constructs on neuronal coding, network dynamics and neural information processing and transmission.
The web-based architecture was chosen to (1) offer a userfriendly interface, (2) reach a wide audience and (3) enable $e$-research and e-learning across the Internet. The modeled circuitry can be totally customized using the user interface.

Additional neuronal structures can be included in the system just running SQL commands into the database. Users interested in making changes and adding developments to the system are invited to download the source code at the system URL (http://remoto.leb.usp.br).

Future work towards increasing the power of the simulator may be visualized within two contexts: improvements of the existing element models and addition of new elements.

In terms of improvements of the mathematical models employed in the simulator, a few examples were already mentioned, as the inclusion of more refined motoneuron and interneuron models. Still another possible improvement with respect to the motoneuron pools are their positioning according to more realistic spatial distributions of lumbosacral motoneurons (Vanderhorst and Holstege 1997; Subramanian et al. 2005). This would include a more randomized placement of each motoneuron along the spinal cord axis (this may be partially attained in the present version of the simulator by choosing a nonzero value for the motoneuron rheobase coefficient of variation in the Miscellaneous panel). Another item that may be improved is that related to the rather rigid stratification of parameter values adopted for the three types of MNs. This may lead to artifacts in simulation results that could be avoided by using a probabilistic approach in the assignment of parameter values among the MN types.

The descending tracts have been modeled in the simulator by spike trains with ISIs having a truncated Gaussian distribution (with selectable mean and standard deviation) or by realizations of a Poisson point process. An interesting extension would be to include gamma-distributed ISIs (Cox and Lewis 1966), as these represent well the positive skewness of the ISI histograms obtained from many real neurons. In addition, gamma-distributed ISIs with orders from 1 to large integers would be able to cover the range from very irregular spike trains (Poisson point process) to very regular ones, the latter with Gaussian-like ISI histograms (central limit theorem).

The models employed in the simulator for motor unit force and EMG generation are simple but computationally very efficient. However, their biological reality could be improved considerably to include effects such as:

1. Sigmoidal relation between force level and MU firing rate (Kernell et al. 1983; Macefield et al. 1996; Van Zandwijk et al. 1996)

2. "Catch" property in slow-type motor units (Burke et al. 1970; Otazu et al. 2001)

3. "Sag" property in fast-type motor units (Burke et al. 1973) 
4. Non-isometric force generation for simulations involving body segment movement (Chin et al. 2006; Siebert et al. 2008)

5. Initiation and extinction of single fiber action potential (at the neuromuscular junction and at tendons, respectively) and its volume conduction to the surface electrodes (Stegeman et al. 2004)

As most of the improvements listed above would require computer-intensive biophysically-based models, their inclusion may be feasible if one simulates a network with a relatively small number of elements or if a more powerful computer is available.

In terms of addition of new elements or features, one direction for future work is the inclusion of models of the muscle spindle and Golgi tendon organ to drive the respective afferent axons that synapse on $\mathrm{Ia}$ and $\mathrm{Ib}$ interneurons (Mileusnic et al. 2006; Mileusnic and Loeb 2006). Another direction is the inclusion of a more complete representation of the interneuronal network of the spinal cord that acts on the different motoneuron pools (Jankowska 1992; Bashor 1998; Pierrot-Deseilligny and Burke 2005).

A comparison of the mammalian spinal cord simulators that have been described in the literature is not an easy task. This is so because it is usually difficult to present in a single paper all the details of the simulator, and also because the simulators are not easily available for testing. Each simulator has its own strong points because its developers were more focused on a specific aspect of the involved physiology or anatomy. For example, the simulator developed by Subramanian et al. (2005) is very strong in the anatomical aspects of the cat spinal cord and in the visualization tools. On the other hand, our simulator seems to have a stronger focus on the neuronal and synaptic physiology and is more oriented towards human electrophysiology. Its interactivity, usability and immediate availability to any neuroscientist connected to the Web are also relevant features.

Acknowledgments This work was supported by grants from Fapesp and $\mathrm{CNP}_{\mathrm{q}}$ (Brazil) to A.F. Kohn. R.R.L. Cisi was a recipient of a fellowship from Fapesp.

\section{References}

Abbott, L. F., Varela, J. A., Sen, K., \& Nelson, S. B. (1997). Synaptic depression and cortical gain control. Science, 275, 220-224.

Andreassen, S., \& Arendt-Nielsen, L. (1987). Muscle fibre conduction velocity in motor units of the human anterior tibial muscle: a new size principle parameter. Journal of Physiology, 391, 561571.

Andreassen, S., \& Rosenfalck, A. (1980). Regulation of the firing pattern of single motor units. Journal of Neurology Neurosurgery and Psychiatry, 43, 897-906.
Ariano, M. A., Armstrong, R. B., \& Edgerton, V. R. (1973). Hindlimb muscle fiber populations of 5 mammals. Journal of Histochemistry \& Cytochemistry, 21, 51-55.

Awiszus, F., \& Feistner, H. (1993). The Relationship between estimates of Ia-Epsp amplitude and conduction-velocity in human soleus motoneurons. Experimental Brain Research, 95, 365-370.

Banks, R. W. (2006). An allometric analysis of the number of muscle spindles in mammalian skeletal muscles. Journal of Anatomy, 208, 753-768.

Barret, J. N., \& Crill, W. E. (1974). Specific membrane properties of cat motoneurons. Journal of Physiology, 239, 301-324.

Bashor, D. P. (1998). A large-scale model of some spinal reflex circuits. Biological Cybernetics, 78, 147-157.

Binder, M. D., Heckman, C. J., \& Powers, R. K. (1996). The physiological control of motoneuron activity. Handbook of physiology. Section 12: Exercise: Regulation and integration of multiple systems pp. 3-53. New York: Oxford University Press.

Booth, V., \& Rinzel, J. (1995). A minimal, compartmental model for a dendritic origin of bistability of motoneuron firing patterns. Journal of Computational Neuroscience, 2, 299-312.

Bower, J. M., Beeman, D., \& Hucka, M. (2003). The GENESIS simulation system. The handbook of brain theory and neural networks pp. 475-478. Cambridge, MA: MIT Press.

Buchthal, F., \& Schmalbruch, H. (1980). Motor unit of mammalian muscle. Physiological Reviews, 60, 90-142.

Bui, T. V., Cushing, S., Dewey, D., Fyffe, R. E., \& Rose, P. K. (2003). Comparison of the morphological and electrotonic properties of Renshaw cells, Ia inhibitory interneurons, and motoneurons in the cat. Journal of Neurophysiology, 90, 2900-2918.

Bui, T. V., Ter-Mikaelian, M., Bedrossian, D., \& Rose, P. K. (2006). Computational estimation of the distribution of L-type $\mathrm{Ca}(2+)$ channels in motoneurons based on variable threshold of activation of persistent inward currents. Journal of Neurophysiology, 95, 225-241.

Burke, R. E. (1981). Motor unit: anatomy, physiology and functional organization. In V. B. Brooks (Ed), Handbook of physiology, The Nervous System. Section II Part I. Bethesda: Am Physiol Soc.

Burke, R. E. (2004). Spinal cord: ventral horn. In G. M. Shepherd (Ed.) The synaptic organization of the brain. New York: Oxford University Press.

Burke, R. E., Levine, D. N., Tsairis, P., \& Zajac, F. E. (1973). Physiological types and histochemical profiles in motor units of cat gastrocnemius. Journal of Physiology, 234, 723-748.

Burke, R. E., Rudomin, P., \& Zajac, F. E. (1970). Catch property in single mammalian motor units. Science, 168, 122-124.

Burke, R. E., Strick, P. L., Kanda, K., Kim, C. C., \& Walmsley, B. (1977). Anatomy of medial gastrocnemius and soleus motor nuclei in cat spinal cord. Journal of Neurophysiology, 40, 667680.

Capaday, C., \& Stein, R. B. (1987). A method for simulating the reflex output of a motoneuron pool. Journal of Neuroscience Methods, 21, 91-104.

Capek, R., \& Esplin, B. (1977). Homosynaptic depression and transmitter turnover in spinal monosynaptic pathway. Journal of Neurophysiology, 40, 95-105.

Carnevale, N. T., \& Hines, M. L. (2006). The NEURON Book. Cambridge: Cambridge University Press.

Carr, P. A., Alvarez, F. J., Leman, E. A., \& Fyffe, R. E. W. (1998). Calbindin D28k expression in immunohistochemically identified Renshaw cells. Neuroreport, 9, 2657-2661.

Chan, K. M., Doherty, T. J., \& Brown, W. F. (2001). Contractile properties of human motor units in health, aging, and disease. Muscle and Nerve, 24, 1113-1133.

Chin, L., Yue, P., Feng, J. J., \& Seow, C. Y. (2006). Mathematical simulation of muscle cross-bridge cycle and force-velocity relationship. Biophysical Journal, 91, 3653-3663. 
Cisi, R. R. L., \& Kohn, A. F. (2004). Spinal cord neuronal network simulator. 28th Conference of the Canadian Medical and Biological Engineering Society, Quebec, Canada.

Cisi, R. R. L., \& Kohn, A. F. (2007). H-reflex depression simulated by a biologically realistic motoneuron network. 29th Annual International Conference of the IEEE Engineering in Medicine and Biology Society, Lyon, France.

Clamann, H. P. (1969). Statistical analysis of motor unit firing patterns in a human skeletal muscle. Biophysical Journal, 9, $1233-1251$.

Cleveland, S., Kuschmierz, A., \& Ross, H. G. (1981). Static inputoutput relations in the spinal recurrent inhibitory pathway. Biological Cybernetics, 40, 223-231.

Cooper, S. (1966). Muscle spindles and motor units. In B. L. Andrew (Ed.) Control and innervation of skeletal muscle (pp. 9-17). Edinburgh: Livingstone.

Cox, D. R., \& Isham, V. (1980). Point processes. London: Chapman and Hall.

Cox, D. R., \& Lewis, P. A. W. (1966). The statistical analysis of series of events. London: Methuen.

Cullheim, S., \& Kellerth, J. O. (1978). Morphological-study of axons and recurrent axon collaterals of cat alpha-motoneurones supplying different functional types of muscle unit. Journal of Physiology, 281, 301-313.

Dai, Y., Jones, K. E., Fedirchuk, B., McCrea, D. A., \& Jordan, L. M. (2002). A modelling study of locomotion-induced hyperpolarization of voltage threshold in cat lumbar motoneurones. Journal of Physiology, 544, 521-536.

Datta, A. K., Farmer, S. F., \& Stephens, J. A. (1991). Central nervous pathways underlying synchronization of human motor unit firing studied during voluntary contractions. Journal of Physiology, 432, 401-425.

De Luca, C. J., Foley, P. J., \& Erim, Z. (1996). Motor unit properties in constant-force isometric contractions. Journal of Neurophysiology, 76, 1503-1516.

De Luca, C. J., \& Forrest, W. J. (1973). Some properties of motor unit action potential trains recorded during constant force isometric contractions in man. Kybernetik, 12, 160-168.

Destexhe, A. (1997). Conductance-based integrate-and-fire models. Neural Computation, 9, 503-514.

Destexhe, A., Mainen, Z. F., \& Sejnowski, T. J. (1994a). An efficient method for computing synaptic conductances based on a kineticmodel of receptor-binding. Neural Computation, 6, 14-18.

Destexhe, A., Mainen, Z. F., \& Sejnowski, T. J. (1994b). Synthesis of models for excitable membranes, synaptic transmission and neuromodulation using a common kinetic formalism. Journal of Computational Neuroscience, 1, 195-230.

Dum, R. P., \& Kennedy, T. T. (1980). Physiological and histochemical-characteristics of motor units in cat tibialis anterior and extensor digitorum longus muscles. Journal of Neurophysiology, 43, 1615-1630.

Feinstein, B., Lindegard, B., Nyman, E., \& Wohlfart, G. (1955). Morphologic studies of motor units in normal human muscles. Acta Anatomica, 23, 127-142.

Finkel, A. S., \& Redman, S. J. (1983). The synaptic current evoked in cat spinal motoneurones by impluses in single group Ia axons. Journal of Physiology, 342, 615-632.

Fleshman, J. W., Segev, I., \& Burke, R. E. (1988). Electrotonic architecture of type-identified alpha-motoneurons in the cat spinal cord. Journal of Neurophysiology, 60, 60-85.

Floeter, M. K., \& Kohn, A. F. (1997). H-Reflex of different sizes exhibit differential sensitivity to low frequency depression. Electroencephalography and Clinical Neurophysiology, 105, 470-475.

Friedman, W. A., Sypert, G. M., Munson, J. B., \& Fleshman, J. W. (1981). Recurrent inhibition in type-identified motoneurons. Journal of Neurophysiology, 46, 1349-1359.
Fuglevand, A. J., Winter, D., \& Patla, A. E. (1993). Models of recruitment and rate coding organization in motor-unit pools. Journal of Neurophysiology, 70, 2470-2488.

Fuglevand, A. J., Winter, D. A., Patla, A. E., \& Stashuk, D. (1992). Detection of motor unit action potentials with surface electrodes: influence of electrode size and spacing. Biological Cybernetics, 67, 143-153.

Garnett, R. A. F., O'Donovan, M. J., Stephens, J. A., \& Taylor, A. (1979). Motor Unit Organization of Human Medial Gastrocnemius. Journal of Physiology, 287, 33-43.

Giugliano, M. (2000). Synthesis of generalized algorithms for the fast computation of synaptic conductances with Markov kinetic models in large network simulations. Neural Computation, 12, 903-931.

Halonen, J. P., Falck, B., \& Kalino, H. (1981). The firing rate of motor units in neuromuscular disorders. Journal of Neurophysiology, $225,269-276$.

Heckman, C.J., Gorassini, M.A., \& Bennett, D.J. (2005). Persistent inward currents in motoneuron dendrites: implications for motor output. Muscle and Nerve, 31, 135-156.

Henneman, E., Somjen, G., \& Carpenter, D. O. (1965). Excitability and inhibitability of motoneurons of different sizes. Journal of Neurophysiology, 28, 599-620.

Hermens, J. H., Baten, C. T. M., Boom, H. B. K., \& Rutten, W. L. C. (1992). Distribution of MUAP amplitude and duration estimated from surface EMG. Proceedings of the 14th Annual International Conference of the IEEE Engineering in Medicine and Biology Society. Paris, France.

Hines, M. L., Eichner, H., \& Schurmann, F. (2008). Neuron splitting in compute-bound parallel network simulations enables runtime scaling with twice as many processors. Journal of Computational Neuroscience (in press).

Hultborn, H., Katz, R., \& Mackel, R. (1988). Distribution of recurrent inhibition within a motor nucleus. II. Amount of recurrent inhibition in motoneurones to fast and slow units. Acta Physiologica Scandinavica, 34, 363-374.

Hultborn, H., \& Pierrot-Deseilligny, E. (1979). Input-output relations in the pathways of recurrent inhibition to motoneurones in the cat. Journal of Physiology, 297, 267-287.

Ivashko, D. G., Prilutsky, B. I., Markin, S. N., Chapin, J. K., \& Rybak, I. A. (2003). Modeling the spinal cord neural circuitry controlling cat hindlimb movement during locomotion. Neurocomputing, $52-4,621-629$.

Jankowska, E. (1992). Interneuronal relay in spinal pathways from proprioceptors. Progress in Neurobiology, 38, 335-378.

Jankowska, E., \& Hammar, I. (2002). Spinal interneurones; how can studies in animals contribute to the understanding of spinal interneuronal systems in man? Brain Research Reviews, 40, $19-28$.

Jimenez, J., Easton, J. K., \& Redford, J. B. (1970). Conduction studies of the anterior and posterior tibial nerves. Archives of Physical Medicine and Rehabilitation, 51, 164-169.

Johnson, M. A., Polgar, J., Weightman, D., \& Appleton, D. (1973). Data on the distribution of fibre types in thirty-six human muscles an autopsy study. Journal of Neurological Sciences, 18 , 111-129.

Kernell, D. (1965). High-frequency repetitive firing of cat lumbosacral motoneurones stimulates by long-lasting injected currents. Acta Physiologica Scandinavica, 65, 74-86.

Kernell, D. (1972). The early phase of adaptation in repetitive impulse discharges of cat spinal motoneurones. Brain Research, 41, 184-186.

Kernell, D. (1986). Organization and properties of spinal motoneurones and motor units. Progress in Brain Research, 64, 21-30.

Kernell, D., Eerbeek, O., \& Verhey, B. A. (1983). Relation between isometric force and stimulus rate in cat's hindlimb motor units of 
different twitch contraction time. Experimental Brain Research, 50, 220-227.

Kernell, D., \& Monster, A. W. (1982). Time course and properties of late adaptation in spinal motoneurones of the cat. Experimental Brain Research, 46, 191-196.

Kohn, A. F., Floeter, M. K., \& Hallet, M. (1995). A model-based approach for the quantification of $\mathrm{H}$ reflex depression in humans. Proceedings of the 17th Annual International Conference of the IEEE Engineering in Medicine and Biology Society, Montreal, Canada.

Kohn, A. F., Floeter, M. K., \& Hallett, M. (1997). Presynaptic inhibition compared with homosynaptic depression as an explanation for soleus H-reflex depression in humans. Experimental Brain Research, 116, 375-380.

Lo Conte, L. R., Merletti, R., \& Sandri, G. V. (1994). Hermite expansions of compact support waveforms: applications to myoelectric signals. IEEE Transactions on Biomedical Engineering, 41, 1147-1159.

Lowery, M. M., \& Erim, Z. (2005). A simulation study to examine the effect of common motoneuron inputs on correlated patterns of motor unit discharge. Journal of Computational Neuroscience, $19,107-124$.

Lytton, W. W. (1996). Optimizing synaptic conductance calculation for network simulations. Neural Computation, 8, 501-509.

Macefield, V. G., Fuglevand, J., \& Bigland-Ritchie, B. (1996). Contractile properties of single motor units in human toe extensor assessed by intraneural motor axon stimulation. Journal of Neurophysiology, 75, 2509-2519.

MacGregor, R. (1987). Neural and brain modeling. New York: Academic

Maganaris, C. N., Baltzopoulos, V., \& Sargeant, A. (1998). In vivo measurement of the triceps surae complex architecture in man: implications for muscle function. Journal of Physiology, 512, 603-614.

Maltenfort, M. G., Heckman, C. J., \& Rymer, W. Z. (1998). Decorrelating actions of Renshaw interneurons on the firing of spinal motoneurons within a motor nucleus: a simulation study. Journal of Neurophysiology, 80, 309-323.

McComas, A. J. (1991). Invited review: motor unit estimation: methods, results and present status. Muscle and Nerve, 14, 585-597.

McCurdy, M. L., \& Hamm, T. M. (1994a). Spatial and temporal features of recurrent facilitation among motoneurons innervating synergistic muscle of the cat. Journal of Neurophysiology, 72, 227-234.

McCurdy, M. L., \& Hamm, T. M. (1994b). Topography of recurrent inhibition postsynaptic potentials between individual motoneurons in the cat. Journal of Neurophysiology, 72, 214-226.

Meunier, S., Kwon, J., Russmann, H., Ravindran, S., Mazzocchio, R., \& Cohen, L. (2007). Spinal use-dependent plasticity of synaptic transmission in humans after a single cycling session. Journal of Physiology, 579, 375-388.

Mileusnic, M. P., Brown, I. E., Lan, N., \& Loeb, G. E. (2006). Mathematical models of proprioceptors. I. Control and transduction in the muscle spindle. Journal of Neurophysiology, 96, $1772-1788$.

Mileusnic, M. P., \& Loeb, G. E. (2006). Mathematical models of proprioceptors. II. Structure and function of the Golgi tendon organ. Journal of Neurophysiology, 96, 1789-1802.

Misiaszek, J. E. (2003). The H-reflex as a tool in neurophysiology: its limitations and uses in understanding nervous system function. Muscle and Nerve, 28, 144-160.

Mochizuki, G., Ivanova, T. D., \& Garland, S. J. (2005). Synchronization of motor units in human soleus muscle during standing postural tasks. Journal of Neurophysiology, 94, 62-69.

Moritz, C. T., Barry, B. K., Pascoe, M. A., \& Enoka, R. M. (2005). Discharge rate variability influences the variation in force fluctuations across the working range of a hand muscle. Journal of Neurophysiology, 93, 2449-2459.

Nussbaumer, R. M., Ruegg, D. G., Studer, L. M., \& Gabriel, J. P. (2002). Computer simulation of the motoneuron pool-muscle complex. I. Input system and motoneuron pool. Biological Cybernetics, 86, 317-333.

Oppenheim, A. V., Schafer, R. W., \& Buck, J. R. (1999). Discretetime signal processing. New Jersey: Prentice Hall.

Otazu, G. H., Futami, R., \& Hoshimiya, N. (2001). A muscle activation model of variable stimulation frequency response and stimulation history, based on positive feedback in calcium dynamics. Biological Cybernetics, 84, 193-206.

Person, R. S., \& Kudina, L. P. (1972). Discharge frequency and discharge pattern of human motor units during voluntary contraction of muscle. Electroencephalography and Clinical Neurophysiology, 32, 471-483.

Pierrot-Deseilligny, E., \& Burke, D. (2005). The circuitry of the human spinal cord. New York: Cambridge University Press.

Plonsey, R. (1974). The active fiber in a volume conductor. IEEE Transactions on Biomedical Engineering, 21, 371-381.

Poliakov, A. V., Miles, T. S., \& Nordstrom, M. A. (1995). Discharge patterns of tonically firing human motoneurons. Biological Cybernetics, 73, 189-194.

Powers, R. K. (1993). A variable-threshold motoneuron model that incorporates time and voltage-dependent potassium and calcium conductances. Journal of Neurophysiology, 70, 246262.

Powers, R. K., Sawczuk, A., Musick, J. R., \& Binder, M. D. (1999). Multiple mechanisms of spike-frequency adaptation in motoneurones. Journal of Physiology - Paris, 93, 101-114.

Rall, W. (1967). Distinguishing theoretical synaptic potentials computed for different soma-dendritic distributions of synaptic input. Journal of Neurophysiology, 30, 1138-1168.

Rall, W., Burke, R. E., Holmes, W. R., Jack, J. J. B., Redman, S. J., \& Segev, I. (1992). Matching dendritic neuron models to experimental-data. Physiological Reviews, 72, S159-S186.

Rosenfalck, A., \& Andreassen, S. (1980). Impaired regulation of force and firing pattern of single motor units in patients with spasticity. Journal of Neurology, Neurosurgery and Psychiatry, 43, 907-916.

Schwindt, P. C., \& Crill, W. E. (1984). Membrane properties of cat spinal motoneurons. Handbook of the Spinal Cord, 2/3, 199242.

Scott, J. G., \& Mendell, L. M. (1976). Individual Epsps produced by single triceps surae Ia afferent-fibers in homonymous and heteronymous motoneurons. Journal of Neurophysiology, 39, 679-692.

Siebert, T., Rode, C., Herzog, W., Till, O., \& Blickhan, R. (2008). Nonlinearities make a difference: comparison of two common Hill-type models with real muscle. Biological Cybernetics, 98, $133-143$

Stegeman, D. F., Merletti, R., \& Hermens, H. J. (2004). EMG modeling and simulation. Electromyography. In R. Merletti, \& P. A. Parker (Eds.) Physiology, engineering and noninvasive applications. Hoboken: Wiley.

Stienen, A. H., Schouten, A. C., Schuurmans, J., \& van der Helm, F. C. (2007). Analysis of reflex modulation with a biologically realistic neural network. Journal of Computational Neuroscience, $23,333-348$.

Stuart, G. J., \& Redman, S. J. (1990). Voltage dependence of Ia reciprocal inhibitory currents in cat spinal motoneurones. Journal of Physiology, 420, 111-125.

Subramanian, K., Okey, P., Miller, M., \& Bashor, D. (2005). NVIZ: An integrated environment for simulation, visualization and analysis of spinal neuronal dynamics. Journal of Imaging Science and Technology, 49, 505-519. 
Taylor, A. M., \& Enoka, R. M. (2004). Quantification of the factors that influence discharge correlation in model motor neurons. Journal of Neurophysiology, 91, 796-814.

Uchiyama, T., Johansson, H., \& Windhorst, U. (2003). A model of the feline medial gastrocnemius motoneuron-muscle system subjected to recurrent inhibition. Biological Cybernetics, 89, $139-151$.

Uchiyama, T., \& Windhorst, U. (2007). Effects of spinal recurrent inhibition on motoneuron short-term synchronization. Biological Cybernetics, 96, 561-575.

Van Zandwijk, J. P., Bobbert, M. F., Baan, G. C., \& Huijing, P. A. (1996). From twitch to tetanus: performance of excitation dynamics optimized for a twitch in predicted tetanic muscle forces. Biological Cybernetics, 75, 409-417.

Vanderhorst, V. G., \& Holstege, G. (1997). Organization of lumbosacral motoneuronal cell groups innervating hindlimb, pelvic floor, and axial muscles in the cat. Journal of Comparative Neurology, 382, 46-76.

Vandervoort, A. A., \& McComas, A. J. (1983). A comparison of the contractile properties of the human gastrocnemius and soleus muscles. European Journal of Applied Physiology and Occupational Physiology, 51, 435-440.
Vieira, M. F., \& Kohn, A. F. (2007). Compartmental models of mammalian motoneurons of types S, FR and FF and their computer simulation. Computers in Biology and Medicine, 37, $842-860$.

Walmsley, B., \& Tracey, D. J. (1981). An intracellular study of Renshaw cells. Brain Research, 223, 170-175.

Windhorst, U. (1990). Activation of Renshaw cells. Progress in Neurobiology, 35, 135-179.

Windhorst, U. (1996). On the role of recurrent inhibitory feedback in motor control. Progress in Neurobiology, 49, 517-587.

Yao, W., Fuglevand, R. J., \& Enoka, R. M. (2000). Motor-unit synchronization increases EMG amplitude and decreases force steadiness of simulated contractions. Journal of Neurophysiology, $83,441-452$.

Zengel, J. E., Reid, S. A., Sypert, G. W., \& Munson, J. B. (1985). Membrane electrical-properties and prediction of motor-unit type of medial gastrocnemius motoneurons in the cat. Journal of Neurophysiology, 53, 1323-1344.

Zhou, P., \& Rymer, W. Z. (2004). MUAP number estimates in surface EMG: template-matching methods and their performance boundaries. Annals of Biomedical Engineering, 32, 10071015 . 